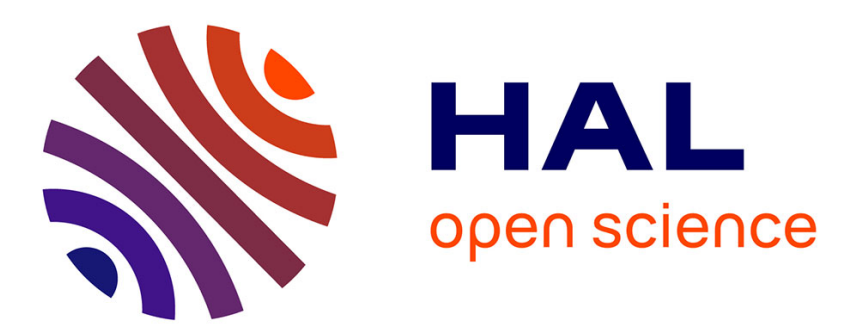

\title{
A general boundary layer corrector for the asymptotic homogenization of elastic linear composite structures
}

Mouad Fergoug, Augustin Parret-Fréaud, Nicolas Feld, Basile Marchand, Samuel Forest

\section{- To cite this version:}

Mouad Fergoug, Augustin Parret-Fréaud, Nicolas Feld, Basile Marchand, Samuel Forest. A general boundary layer corrector for the asymptotic homogenization of elastic linear composite structures. Composite Structures, 2022, pp.115091. 10.1016/j.compstruct.2021.115091 . hal-03531347

\section{HAL Id: hal-03531347 https://hal.science/hal-03531347}

Submitted on 18 Jan 2022

HAL is a multi-disciplinary open access archive for the deposit and dissemination of scientific research documents, whether they are published or not. The documents may come from teaching and research institutions in France or abroad, or from public or private research centers.
L'archive ouverte pluridisciplinaire HAL, est destinée au dépôt et à la diffusion de documents scientifiques de niveau recherche, publiés ou non, émanant des établissements d'enseignement et de recherche français ou étrangers, des laboratoires publics ou privés. 


\title{
A general boundary layer corrector for the asymptotic homogenization of elastic linear composite structures
}

\author{
Mouad Fergoug $^{1,2 *}$, Augustin Parret-Fréaud ${ }^{1}$, Nicolas Feld $^{1}$, Basile Marchand ${ }^{2}$, \\ Samuel Forest ${ }^{2}$ \\ ${ }^{1}$ Safran Tech, Etablissement Paris Saclay, rue des Jeunes Bois-Châteaufort, 78114 \\ Magny-les-Hameaux, France \\ ${ }^{2}$ MINES ParisTech, PSL University, MAT - Centre des matériaux, CNRS UMR 7633, BP 87 \\ 91003 Evry, France
}

\begin{abstract}
Asymptotic homogenization method is often used in multiscale analysis of periodic structures instead of conducting a full field heterogeneous analysis, in order to achieve computational feasibility and efficiency. When completed with a relocalization process, this method may provide relevant estimates to microscale fields within the material. Nevertheless, the construction of a solution near the boundaries remains beyond the capabilities of classical relocalization schemes due to the loss of periodicity in the vicinity of the boundaries. This paper proposes a post-processing scheme in order to conduct the relocalization step within a finite element framework for periodic linear elastic composite materials. It also assesses the boundary layer effect and a new general method, effective for various boundary conditions (Dirichlet, Neumann or mixed), is proposed based on the idea of computing corrective terms as solution of auxiliary problems on the unit-cell. These terms are finally added to the usual fields obtained from the relocalization process to obtain the corrected solution near the boundaries. The efficiency, accuracy and limitation of the proposed approach are studied on various numerical examples.
\end{abstract}

Keywords: Composites structures, Periodic media, Asymptotic homogenization, Boundary layer corrector, Edge effect.

\footnotetext{
${ }^{*}$ Corresponding author

Email address: mouad.fergoug@mines-paristech.fr (Mouad Fergoug ${ }^{1,2}$ )
} 


\section{Introduction}

Composite materials microstructure can be varied, ranging from randomly distributed phases to a perfectly periodic microstructure. Direct Numerical Simulations (DNS, i.e. when the geometry of the microstructure is explicitly described in simulations) of composite materials are often difficult to perform because of the resulting complexity and size of the computational problems. Therefore, to fully realize the benefits offered by these materials, it is essential to develop reliable computational methods, bypassing DNS, to predict their behavior.

Homogenization of periodic structures has been successfully used to determine their effective properties at macroscale from the knowledge of local mechanical properties of one unit-cell, representative of the material microstructure. The obtained effective properties can then be used for numerical simulations of the homogeneous problem without conducting DNS. The unit-cell problem can be solved either analytically or numerically.

The simplest analytical methods are based on the assumption that either strains or stresses are uniform within the unit-cell, leading to the well known Voigt [1] and Reuss [2] bounds. More accurate analytical methods were developed, among which the composite sphere model and the self-consistent scheme $[3,4,5]$. Numerical homogenization using computational methods like the Finite Element Method (FEM) has also been used for solving the unit-cell problem $[6,7,8]$, among many others.

Most of these methods are known to be effective for materials with large scale separation between the scale of heterogeneity and the macroscale dimension. For low scale separation, however, they generally become inaccurate. In such a case, the wave length of variation of the macroscale fields is not sufficiently large compared to the size of the heterogeneities. Thus, the predicted effective properties may fail to describe the local or global response of the composite [9]. To tackle this, higher-order gradients of macroscale strain may be taken into account in the homogenization of the unit-cell. At least two approaches exist regarding this subject:

- The first approach uses Quadratic Boundary Conditions (QBCs) applied to the unit-cell [10, 11, 12], deduced from the macroscale higher-order strain-stress fields. This method has a major flaw as shown in [12]. Indeed, the effective strain-gradient properties remain non zero when the material is homogeneous, which seems to be physically unreasonable result. To tackle this, a correction has been proposed in [13] by adding adequate body forces to QBCs.

- The second approach considers higher-order problems in the asymptotic expansion at the basis of homogenization method, introduced in [14, 15]. This 
method consists in using asymptotic expansions of mechanical fields of the microscale problem in order to split them into separate microscale and macroscale problems. It is shown in [16] that the higher-order terms in asymptotic homogenization introduce successive gradients of macroscale strain and tensors, characteristic of the microstructure, which result in introducing a non-local effect in the material behavior.

These methods allow the prediction of both the local, by a relocalization process, and the overall averaged properties of the structure. A recent comparison between the asymptotic homogenization and QBCs-based method [17] shows that a modification of the QBCs-based method, by adding body forces, is necessary to be consistent with the asymptotic homogenization.

Asymptotic homogenization has been applied in a wide range of engineering problems, e.g. to optimize structures $[18,19,20]$, compute the effective elastic behavior of woven fabric composites [21] and evaluate localized stiffness degradation [22]. Different numerical methods have been applied in conjunction with the asymptotic homogenization theory. The Fast Fourier Transform [23, 24] (FFT) method was used to apply the homogenization theory on microstructures defined on regular grids [25]. FEM has also been successfully applied for the analysis of linear and non-linear microstructures with arbitrary discretization, albeit at a higher computing cost. Different implementation strategies [26, 27, 28, 29] were proposed for the homogenization of composites using the FEM. However, the validity of the relocalization process is usually not verified, except asymptotically in an idealized setting. The analysis of local gradients and boundary effects requires additional efforts.

The response of a composite structure near its geometric boundaries has been studied both experimentally and analytically $[30,31,32,33]$. It has been shown that complex stress states with a rapid change of gradients occur within a very local region near the boundaries, frequently referred to as a boundary layer effect. Ultimately, the high stresses developed in these regions may be responsible for the failure initiation of the structure [30].

While asymptotic homogenization allows to estimate local fields within the structure by a relocalization method, the construction of a solution near the vicinity of the boundaries remains beyond capabilities of the classical homogenization [34] for two reasons:

- First, in asymptotic homogenization, the solution is considered to be periodic. However the loads are no longer periodic in the vicinity of boundaries, and consequently, the solution is not periodic either.

- Second, the boundary condition on the composite domain cannot be satisfied 
by a periodic unit-cell solution.

One approach to tackle the non-periodicity in the boundary layers is to introduce evanescent corrective terms that would exponentially decay toward the interior of the body $[35,36]$. These corrective terms are obtained by solving auxiliary problems on the unit-cell. An application of this approach is found in [37], in which heterogeneous stress fields are estimated by considering a Neumann boundary condition correction as in [35]. More recently, a numerical study was conducted to investigate the decay of the boundary layer in a three dimensional periodic homogenization for different fiber orientations using a domain with fixed edges (Dirichlet boundary) [38]. It is worth to remark that these papers have not proposed a boundary layer correction strategy for various types of boundary conditions.

Regarding the aforementioned aspects, the present work proposes a general boundary layer correction methodology for asymptotic homogenization in order to approximate real microscale fields near the boundaries. The main idea is to compute corrective terms obtained from the resolution of different problems over the unit-cell. The nature of the problems to be solved depends on the actual boundary conditions applied locally to the structure. Then, the corrective terms are added to the estimated local fields. The obtained results demonstrate the significance of boundary layer corrections even in the general case. Indeed, the proposed method is valid for different Boundary Conditions (BCs): Dirichlet, Neumann or mixed. The authors are unaware of a similar general boundary layer correction strategy for asymptotic homogenization in the literature.

The outline of the paper is as follows. In Sec. 2, we first recall briefly the asymptotic expansion homogenization method and describe the proposed relocalization process. In Sec. 3, we detail the proposed general boundary layer correction procedure. Numerical examples are presented and discussed in Sec. 4, with the objective of demonstrating the efficiency and limitations of the suggested approach.

\section{Estimation of microscale fields based on asymptotic homogenization}

This section briefly recalls the asymptotic homogenization approach in linear elasticity. The reader is referred to seminal works in $[14,15]$ and other recent references such as $[16,26,39]$ for further details. We also describe the proposed estimation of microscale fields based on the relocalization process, derived from asymptotic homogenization. This relocalization stage is associated with a given macroscale equilibrium state detailed in this section. 


\subsection{Statement of the boundary value problem and homogenization procedure}

An inhomogeneous body is considered as a linearly elastic solid in static equilibrium, whose heterogeneity arises from the distribution of separate phases at the microscale. We define the bounded domain $\Omega^{\epsilon}$ occupied by this heterogeneous body and corresponding to the microscale (see Fig. 1) and subjected to a body force $\boldsymbol{f}$ per unit volume. The boundary $\partial \Omega^{\epsilon}$ consists of a portion $\Gamma_{u}$, on which the displacements are prescribed to the value $\boldsymbol{u}^{d}$, and a portion $\Gamma_{t}$ on which surface tractions $\boldsymbol{F}^{d}$ per unit area are prescribed such that $\partial \Omega^{\epsilon}=\Gamma_{u} \cup \Gamma_{t}$, and $\Gamma_{u} \cap \Gamma_{t}=\emptyset$.

Due to its heterogeneity, the mechanical behavior of the body is assumed to depend on two scales:

- Macroscale, free of heterogeneities, having $L$ as a characteristic length and global coordinates $\boldsymbol{x} \in \Omega$ with the assumption that $\partial \Omega^{\epsilon}=\partial \Omega$ (see Fig. 1).

- Microscale, having $l$ as a characteristic length and with local coordinates $\boldsymbol{y} \in Y$, where $Y$ is the unit-cell domain, typically chosen to be an open rectangular parallelepiped $Y=] 0, Y_{1}[\times] 0, Y_{2}[\times] 0, Y_{3}[$ (see Fig. 1).

The coarse and fine scales are related by the parameter $\epsilon$ such that:

$$
\epsilon=\frac{l}{L} \quad \boldsymbol{y}=\frac{\boldsymbol{x}}{\epsilon}
$$

The domain $\Omega^{\epsilon}$ can be considered as the product space $\Omega \times Y$ :

$$
\Omega^{\epsilon}=\left\{\boldsymbol{x} \in \Omega \mid\left(\boldsymbol{y}=\frac{\boldsymbol{x}}{\epsilon}\right) \in Y\right\}
$$

We further consider that, at each material point of $\Omega^{\epsilon}$, there exists a periodically repeating microstructure. Owing to this periodicity, one can define the elasticity tensor $\mathbb{C}$ as $Y$-periodic:

$$
\mathbb{C}=\mathbb{C}(\boldsymbol{y})
$$

Expressed in global coordinates, the heterogeneous stiffness tensor would read $\mathbb{C}^{\epsilon}(\boldsymbol{x})=$ $\mathbb{C}(\boldsymbol{x} / \epsilon)=\mathbb{C}(\boldsymbol{y})$, the superscript indicating fine-scale dependence. Similarly, the microscale displacement, strain and stress fields in global coordinates read $\boldsymbol{u}^{\epsilon}, \varepsilon^{\epsilon}$, and $\boldsymbol{\sigma}^{\epsilon}$, respectively.

In the ensuing sections, we will define the following boundary value problems:

- The heterogeneous problem $\left(\mathcal{P}_{\text {ref }}\right)$ corresponding to microscale and generated by translating the unit-cell $Y$ characterized by an oscillatory behavior $\mathbb{C}^{\epsilon}(\boldsymbol{x})$ over the three-space directions. 


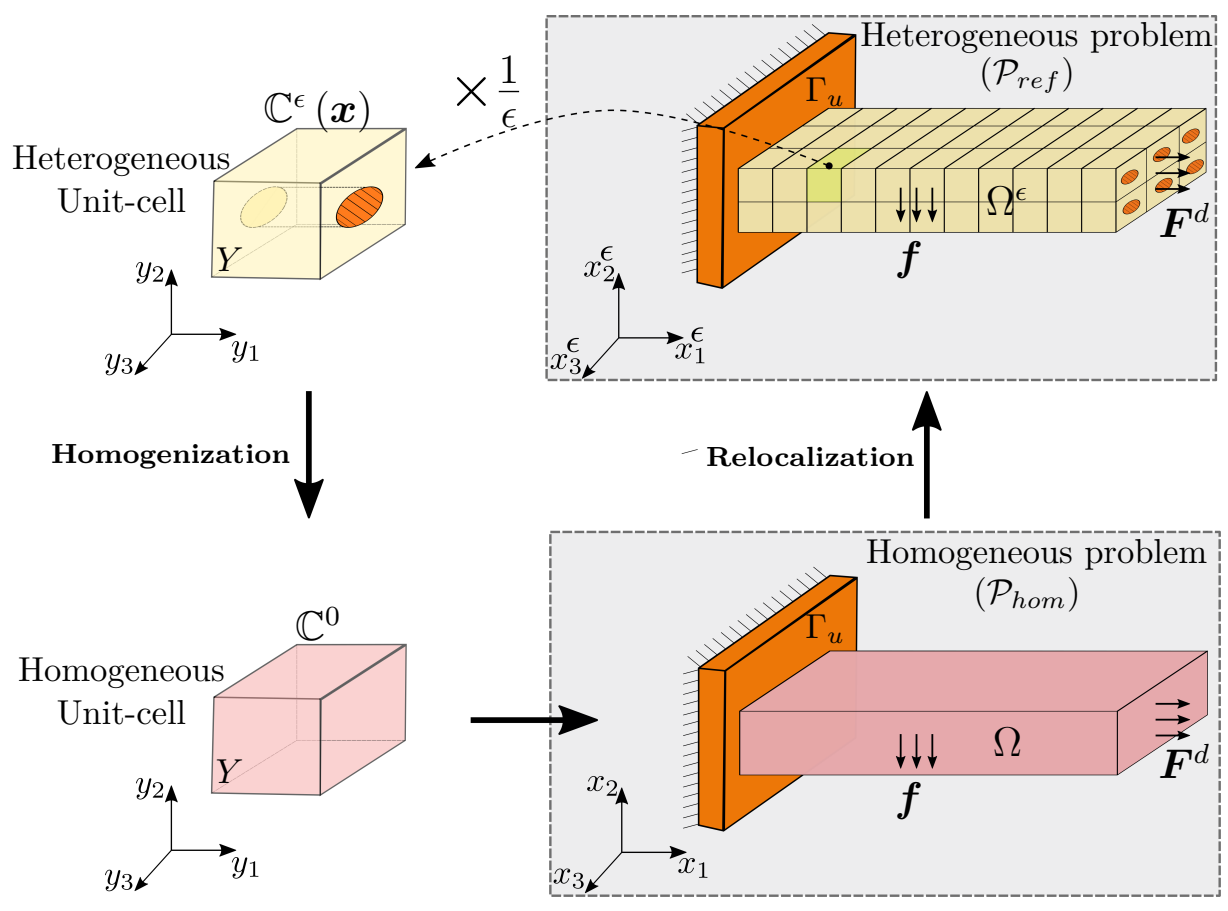

Figure 1: Illustration of the heterogeneous problem $\left(\mathcal{P}_{\text {ref }}\right)$ with domain $\Omega^{\epsilon}$, constructed by translating the unit-cell $Y$ characterized by an oscillatory behavior $\mathbb{C}^{\epsilon}(\boldsymbol{x})$ over the three-space directions. The homogenized problem $\left(\mathcal{P}_{\text {hom }}\right)$ with homogeneous domain $\Omega$ is characterized by the homogenized elasticity tensor $\mathbb{C}^{0}$ obtained from the homogenization step. Microscale fields are estimated from a relocalization process.

- The first-order periodic problem $\left(\mathcal{P}_{\text {order }}^{1^{s t}}\right)$ used to deduce the homogenized elasticity tensor $\mathbb{C}^{0}$ and localization tensors.

- The homogenized problem $\left(\mathcal{P}_{\text {hom }}\right)$ corresponding to the macroscale characterized by the homogenized elasticity tensor $\mathbb{C}^{0}$.

The proposed first-order estimate of microscale fields will also be defined.

Definition of the heterogeneous problem $\left(\mathcal{P}_{\text {ref }}\right)$

The boundary value problem of the heterogeneous linear elastic body $\Omega^{\epsilon}$ reads: 
Find $\left(\boldsymbol{u}^{\epsilon}, \boldsymbol{\sigma}^{\epsilon}\right)$ such that:

$$
\begin{cases}\operatorname{div}\left(\boldsymbol{\sigma}^{\epsilon}(\boldsymbol{x})\right)+\boldsymbol{f}(\boldsymbol{x})=0 & \forall \boldsymbol{x} \in \Omega^{\epsilon} \\ \boldsymbol{\sigma}^{\epsilon}(\boldsymbol{x})=\mathbb{C}^{\epsilon}(\boldsymbol{x}): \boldsymbol{\varepsilon}^{\epsilon}(\boldsymbol{x}) & \forall \boldsymbol{x} \in \Omega^{\epsilon} \\ \boldsymbol{u}^{\epsilon}(\boldsymbol{x})=\boldsymbol{u}^{d} & \forall \boldsymbol{x} \in \Gamma_{u} \\ \boldsymbol{\sigma}^{\epsilon}(\boldsymbol{x}) \cdot \boldsymbol{n}=\boldsymbol{F}^{d} & \forall \boldsymbol{x} \in \Gamma_{t}\end{cases}
$$

where Eq. (4a) is the balance equation, Eq. (4b) is the constitutive equation and Eq. (4c), Eq. (4d) are the applied boundary conditions, with $\boldsymbol{n}$ the outward unit normal vector to $\Gamma_{t}$. The traction and displacement fields are assumed to be continuous across the interfaces. The strain field $\varepsilon^{\epsilon}$ is given by:

$$
\varepsilon^{\epsilon}=\operatorname{sym}\left(\nabla \boldsymbol{u}^{\epsilon}\right)=\frac{1}{2}\left(\nabla \boldsymbol{u}^{\epsilon}+\left(\nabla \boldsymbol{u}^{\epsilon}\right)^{\top}\right)
$$

where $\operatorname{sym}(\bullet)$ indicates the symmetric part of second-order tensors.

Thus, by considering Einstein's convention for tensor notation, the resolution of $\left(\mathcal{P}_{\text {ref }}\right)$ consists in determining the displacement field corresponding to the solution $\boldsymbol{u}^{\epsilon}$ of the following variational problem:

$$
\int_{\Omega^{\epsilon}} C_{i j k l} \frac{\partial u_{k}^{\epsilon}}{\partial x_{l}^{\epsilon}} \frac{\partial v_{i}}{\partial x_{j}^{\epsilon}} d \Omega^{\epsilon}=\int_{\Omega^{\epsilon}} f_{i} v_{i} d \Omega^{\epsilon}+\int_{\Gamma_{t}} F_{i}^{d} v_{i} d \Gamma \quad \forall v_{i} \in \mathcal{V}_{\Omega^{\epsilon}}^{0}
$$

where $\mathcal{V}_{\Omega^{\epsilon}}^{0}$ are the sets of sufficiently regular functions, zero-valued in $\Gamma_{u}$. Mechanical fields, solution of $\left(\mathcal{P}_{\text {ref }}\right)$, are approximated with an asymptotic expansion in powers of the small parameter $\epsilon$ as:

$$
\begin{aligned}
& \boldsymbol{u}^{\epsilon}(\boldsymbol{x})=\boldsymbol{u}^{0}(\boldsymbol{x}, \boldsymbol{y})+\epsilon \boldsymbol{u}^{1}(\boldsymbol{x}, \boldsymbol{y})+\mathcal{O}\left(\epsilon^{2}\right) \\
& \varepsilon^{\epsilon}(\boldsymbol{x})=\boldsymbol{\varepsilon}^{0}(\boldsymbol{x}, \boldsymbol{y})+\epsilon \boldsymbol{\varepsilon}^{1}(\boldsymbol{x}, \boldsymbol{y})+\mathcal{O}\left(\epsilon^{2}\right) \\
& \boldsymbol{\sigma}^{\epsilon}(\boldsymbol{x})=\boldsymbol{\sigma}^{0}(\boldsymbol{x}, \boldsymbol{y})+\epsilon \boldsymbol{\sigma}^{1}(\boldsymbol{x}, \boldsymbol{y})+\mathcal{O}\left(\epsilon^{2}\right)
\end{aligned}
$$

The quantities $\boldsymbol{u}^{n}, \boldsymbol{\varepsilon}^{n}$ and $\boldsymbol{\sigma}^{n}$ are $Y$-periodic functions called correctors of order $n$ of the displacement, strain and stress fields, respectively.

The existence of two scales means that a function $f^{\epsilon}(\boldsymbol{x})$ is associated with the function $f(\boldsymbol{x}, \boldsymbol{y})$, where the chain rule is applied:

$$
f^{\epsilon}(\boldsymbol{x})=f(\boldsymbol{x}, \boldsymbol{y}) ; \quad \frac{\mathrm{d}}{\mathrm{d} x} f^{\epsilon}=\frac{\partial}{\partial x} f+\frac{1}{\epsilon} \frac{\partial}{\partial y} f
$$


The expansion of strains is obtained by substituting the expansion of displacements (7) into the strain definition (5), and making use of the chain rule (10). The expansion of stresses is obtained by substituting the resulting strains into the constitutive equation (4b). The stress expansion is substituted into the balance equation (4a) and, by collecting the terms of like powers of $\epsilon$, we obtain a sequence of equilibrium equations of different orders with respect to powers of $\epsilon$, defined on the unit-cell [16]. We choose to ignore higher-order homogenization problems and restrict our study to the resolution of the first-order problem. It is shown [16] that $\boldsymbol{u}^{0}(\boldsymbol{x}, \boldsymbol{y})=\boldsymbol{u}^{0}(\boldsymbol{x})$, meaning that the displacement $\boldsymbol{u}^{0}$ is independent of the microscale coordinates $\boldsymbol{y}$ and can be identified with the macroscale displacement field.

First-order periodic problem $\left(\mathcal{P}_{\text {order }}^{1^{\text {st }}}\right)$

This problem is defined on the unit-cell $Y$. Its solution is the first-order displacement corrector $\boldsymbol{u}^{1}$ and stress $\boldsymbol{\sigma}^{0}$. It reads:

Find $\left(\boldsymbol{u}^{1}, \boldsymbol{\sigma}^{0}\right)$ such that:

$$
\left\{\begin{array}{lrc}
\operatorname{div}_{y}\left(\boldsymbol{\sigma}^{0}(\boldsymbol{x}, \boldsymbol{y})\right)=0 & \forall \boldsymbol{y} \in Y \quad(11 \mathrm{a}) \\
\boldsymbol{\sigma}^{0}(\boldsymbol{x}, \boldsymbol{y})=\mathbb{C}(\boldsymbol{y}):\left(\boldsymbol{E}^{0}(\boldsymbol{x})+\boldsymbol{\varepsilon}_{y}\left(\boldsymbol{u}^{1}\right)\right) & \forall \boldsymbol{y} \in Y \quad(11 \mathrm{~b}) \\
\boldsymbol{u}^{1}(\boldsymbol{x}, \boldsymbol{y}) & \text { is } Y-\text { periodic } & (11 \mathrm{c}) \\
\boldsymbol{\sigma}^{0}(\boldsymbol{x}, \boldsymbol{y}) \cdot \boldsymbol{n} & \text { is } Y-\operatorname{antiperiodic} & (11 \mathrm{~d})
\end{array}\right.
$$

where $\boldsymbol{E}^{0}(\boldsymbol{x})=\boldsymbol{\varepsilon}_{x}\left(\boldsymbol{u}^{0}(\boldsymbol{x})\right)$ is a prescribed macroscale strain, $\operatorname{div}_{y}(\bullet)$ is the divergence operator with respect to local variable $\boldsymbol{y}$, and $\boldsymbol{\varepsilon}_{x}, \boldsymbol{\varepsilon}_{y}$ are the strain tensors calculated according to the global variable $\boldsymbol{x}$ and local variable $\boldsymbol{y}$, respectively:

$$
\varepsilon_{\bullet}=\operatorname{sym}\left(\nabla \cdot \boldsymbol{u}^{\epsilon}\right)=\frac{1}{2}\left(\nabla \cdot \boldsymbol{u}^{\epsilon}+\left(\nabla \cdot \boldsymbol{u}^{\epsilon}\right)^{\top}\right)
$$

The periodic fluctuation solution of the first-order problem takes the following form [16]:

$$
\boldsymbol{u}^{1}(\boldsymbol{x}, \boldsymbol{y})=\mathbb{D}(\boldsymbol{y}): \boldsymbol{E}^{0}(\boldsymbol{x})
$$

where $\mathbb{D}(\boldsymbol{y})$ is a third-order tensor, called the displacement relocalization tensor. It is periodic over unit-cell $Y$ and verifies $\langle\mathbb{D}\rangle_{Y}=0$, where $\langle\bullet\rangle_{Y}=\frac{1}{|Y|} \int_{Y} \bullet d Y$ indicates the volume average over unit-cell $Y$. Note that $\mathbb{D}$ is symmetric with respect to its two last indices. 
The displacement relocalization tensor components are the solutions $D_{i j k} \in \mathcal{V}_{Y}^{\#}$ of the variational problem:

$$
\int_{Y} C_{i j k l} \frac{\partial D_{k m n}}{\partial y_{l}} \frac{\partial v_{i}}{\partial y_{j}} d Y=\int_{Y} C_{i j m n} \frac{\partial v_{i}}{\partial y_{j}} d Y, \quad \forall v_{i} \in \mathcal{V}_{Y}^{\#}
$$

where $\mathcal{V}_{Y}^{\#}$ is the set of $Y$-periodic sufficiently regular functions with zero average value in $Y$.

The total first-order microscale strain field reads:

$$
\boldsymbol{\varepsilon}^{0}(\boldsymbol{x}, \boldsymbol{y})=\boldsymbol{\varepsilon}_{y}\left(\boldsymbol{u}^{1}(\boldsymbol{x}, \boldsymbol{y})\right)+\boldsymbol{E}^{0}(\boldsymbol{x})=\mathbb{A}(\boldsymbol{y}): \boldsymbol{E}^{0}(\boldsymbol{x})
$$

where $\mathbb{A}(\boldsymbol{y})$ is a fourth-order tensor, called the strain relocalization tensor:

$$
\mathbb{A}(\boldsymbol{y})=\mathbb{I}+\varepsilon_{y}(\mathbb{D}(\boldsymbol{y}))
$$

where $\mathbb{I}$ is the fourth-order identity tensor operating on symmetric second order tensors.

We therefore can define the first-order microscale stress field as:

$$
\boldsymbol{\sigma}^{0}(\boldsymbol{x}, \boldsymbol{y})=\mathbb{C}(\boldsymbol{y}): \varepsilon^{0}(\boldsymbol{x}, \boldsymbol{y})=\mathbb{B}(\boldsymbol{y}): \boldsymbol{E}^{0}(\boldsymbol{x})
$$

where

$$
\mathbb{B}(\boldsymbol{y})=\mathbb{C}(\boldsymbol{y}): \mathbb{A}(\boldsymbol{y})
$$

is the stress relocalization tensor. Note that $\mathbb{A}$ and $\mathbb{B}$ possess the minor symmetries. The homogenized elasticity tensor $\mathbb{C}^{0}$ is deduced from the volume average of the stress relocalization tensor over the unit-cell:

$$
\mathbb{C}^{0}=\langle\mathbb{B}(\boldsymbol{y})\rangle_{Y}
$$

It can be proved that $\mathbb{C}^{0}$ possesses the minor and major symmetries as required for an elasticity tensor.

Definition of the homogeneous problem $\left(\mathcal{P}_{\text {hom }}\right)$

The boundary value problem on the macroscale for the homogeneous linear elastic body $\Omega$ reads:

Find $(\boldsymbol{U}, \boldsymbol{\Sigma})$ such that:

$$
\begin{cases}\operatorname{div}_{x}(\boldsymbol{\Sigma}(\boldsymbol{x}))+\boldsymbol{f}(\boldsymbol{x})=0 & \forall \boldsymbol{x} \in \Omega \\ \boldsymbol{\Sigma}(\boldsymbol{x})=\mathbb{C}^{0}: \boldsymbol{E}(\boldsymbol{x}) & \forall \boldsymbol{x} \in \Omega \\ \boldsymbol{U}(\boldsymbol{x})=\boldsymbol{u}^{d} & \forall \boldsymbol{x} \in \boldsymbol{\Gamma}_{u} \\ \boldsymbol{\Sigma}(\boldsymbol{x}) \cdot \boldsymbol{n}=\boldsymbol{F}^{d} & \forall \boldsymbol{x} \in \boldsymbol{\Gamma}_{t}\end{cases}
$$


where $\operatorname{div}_{x}(\bullet)$ is the divergence operator with respect to global variable $\boldsymbol{x}$, the macroscale displacement is $\boldsymbol{U}=\boldsymbol{u}^{0}$ and the macroscale strain $\boldsymbol{E}$ and stress $\boldsymbol{\Sigma}$ are equal to the volume average over the unit-cell of their microscopic counterparts $\varepsilon^{0}$ and $\boldsymbol{\sigma}^{0}$, respectively.

$$
\boldsymbol{E}=\left\langle\varepsilon^{0}\right\rangle_{Y}, \quad \boldsymbol{\Sigma}=\left\langle\boldsymbol{\sigma}^{0}\right\rangle_{Y}
$$

The resolution of $\left(\mathcal{P}_{\text {hom }}\right)$ consists in finding the displacement field corresponding to the solution $\boldsymbol{u}^{0}$ of the following variational problem:

$$
\int_{\Omega} C_{i j k l}^{0} \frac{\partial u_{k}^{0}}{\partial x_{l}} \frac{\partial v_{i}}{\partial x_{j}} d \Omega=\int_{\Omega} f_{i} v_{i} d \Omega+\int_{\Gamma_{t}} F_{i}^{d} v_{i} d \Gamma \quad \forall v_{i} \in \mathcal{V}_{\Omega}^{0}
$$

where $\mathcal{V}_{\Omega}^{0}$ is the set of sufficiently regular functions, zero-valued in $\Gamma_{u}$.

It is apparent from Eq. (20c) and (20d) that macroscale fields $(\boldsymbol{U}, \boldsymbol{\Sigma})$ verify given boundary conditions. However, as we will demonstrate later in this article, the microscale fields estimated after relocalization process, do not necessary verify these boundary conditions. For instance, the estimated local stresses $\boldsymbol{\sigma}^{0}$ do not verify in general the Neumann boundary conditions:

$$
\boldsymbol{\sigma}^{0} \cdot \boldsymbol{n} \neq \boldsymbol{F}^{d}
$$

\subsection{Proposed first-order estimate}

The design and reliability analysis of composite structures taking into account the state of microscale fields can be more accurate than an entirely macroscale analysis. To tackle this, it is possible to bind the macroscale with the microscale based on a relocalization process derived from asymptotic homogenization.

As mentioned before, periodic homogenization is based on the assumption of scale separation between that of heterogeneities (with local coordinates $\boldsymbol{y}$ ) and the characteristic macroscale dimension (with global coordinates $\boldsymbol{x}$ ). This assumption induces a local invariance by translation and the periodicity of microscale fields. The scale separation assumption leads to:

$$
\epsilon \ll 1
$$

with $\epsilon$ defined in Eq. (1). In what follows, we propose to investigate situations where the scale separation assumption is not verified, meaning that the size of the heterogeneities can be comparable to the characteristic macroscale length scale, e.g. near the boundaries. Thus, the macroscale gradients could be such that it is no longer possible to assume a uniform mechanical field acting on the unit-cell. Therefore, we will consider:

$$
\epsilon=1 \Longrightarrow \boldsymbol{x}=\boldsymbol{y}
$$


This directly implies that the separation of scales assumption is not verified. Therefore, the mechanical fields of the reference problem depend only on $\boldsymbol{x}$, representing both microscale and macroscale coordinates.

For the purpose of this study, the reference problem is tractable by Direct Numerical Simulation (DNS) and its mechanical fields are used as reference results. However, in the more general case where a DNS would be out of reach, common practice would call for a best estimate. This estimate is built here using the solution of the homogeneous problem $\left(\mathcal{P}_{\text {hom }}\right)$ and relocalization tensors $(\mathbb{D}, \mathbb{A}, \mathbb{B})$ obtained after the resolution of $\left(\mathcal{P}_{\text {order }}^{\text {st }}\right)$. The proposed estimate fields read:

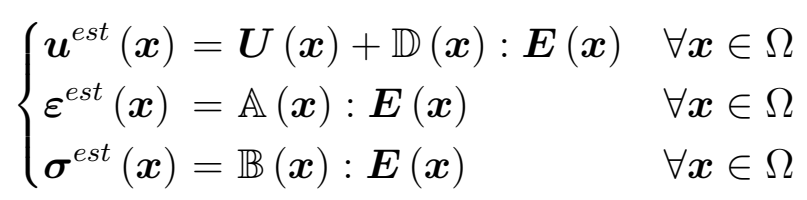

The displacement $\boldsymbol{U}(\boldsymbol{x})$ and the strain $\boldsymbol{E}(\boldsymbol{x})$ are obtained from the resolution of the homogeneous problem $\left(\mathcal{P}_{\text {hom }}\right)$.

\subsection{Proposed relocalization procedure}

Once $\left(\mathcal{P}_{\text {order }}^{1^{\text {st }}}\right)$ is solved, the resulting homogenized elasticity tensor $\mathbb{C}^{0}$ is used to compute the homogeneous structure problem conducted at the macroscale, whose solution fields are $(\boldsymbol{U}, \boldsymbol{E}, \boldsymbol{\Sigma})$. In order to carry out the relocalization process, an intermediate step is performed to locate each unit-cell on the macroscale structure. To eliminate mesh-sensitivity and facilitate the computation of error estimates, we considered that the macroscale mesh was constructed by translating the unit-cell mesh over the three space directions, i.e. macroscale and microscale meshes are identical. If not, a mapping of the homogeneous fields on the microscale mesh could be considered as shown in [40]. We considered the relocalization process improvement proposed in Ref. [41], which is a simple way of taking into account macroscale gradients in relocalization formulation. For instance, the microscopic estimated strain (or stress), in a given point of the unit-cell, is determined using relocalization tensors combined with the value of the current macroscale strain at this point, and not its average over the unit-cell.

\section{Boundary layer correction}

Asymptotic homogenization may provide an estimate of the microscale fields within the material if the macroscale computation is complemented by a relocalization process. Nevertheless, the construction of a solution near the boundaries remains 


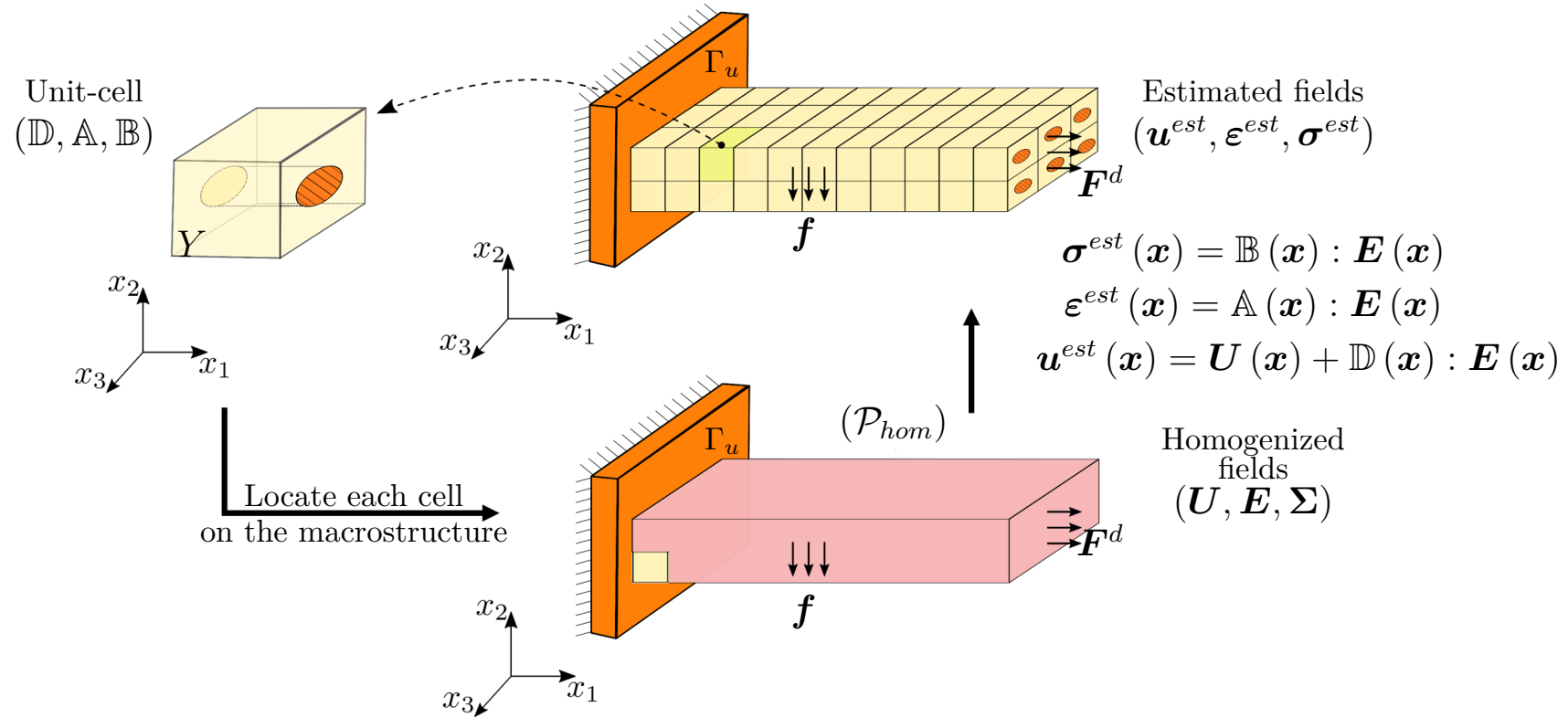

Figure 2: Illustration of the proposed relocalization procedure scheme without scale separation. Mechanical fields depend only on $\boldsymbol{x}$, representing both microscale and macroscale coordinates.

beyond the capabilities of classic homogenization/relocalization. This is mainly due to the loss of periodicity in the vicinity of boundaries.

We propose a new approach to account for non-periodicity in the boundary layers based on the idea of introducing corrective terms that would decay as one moves toward the interior of the body, which can be seen as an extension of $[35,38]$.

The main objective of this section is to describe the proposed general boundary layer correction procedure, effective for various types of boundary conditions (Dirichlet, Neumann, or mixed), see Fig. 3. 


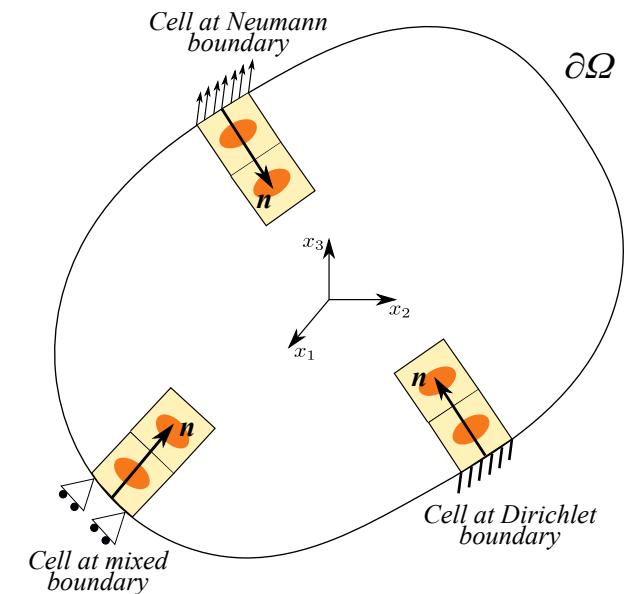

Figure 3: Illustration of the boundary layers for Dirichlet, Neumann, and mixed boundary conditions. With $\boldsymbol{n}$ is the normal direction of the boundary.

\subsection{Correctors for Neumann BCs}

The homogenized problem $\left(\mathcal{P}_{\text {hom }}\right)$ could be written as:

$$
\begin{cases}\operatorname{div}\left\langle\boldsymbol{\sigma}^{e s t}(\boldsymbol{x})\right\rangle_{Y}+\boldsymbol{f}(\boldsymbol{x})=0 & \forall \boldsymbol{x} \in \boldsymbol{\Omega} \\ \left\langle\boldsymbol{\sigma}^{e s t}(\boldsymbol{x})\right\rangle_{Y}=\mathbb{C}^{0}: \boldsymbol{E}(\boldsymbol{x}) & \forall \boldsymbol{x} \in \boldsymbol{\Omega} \\ \left\langle\boldsymbol{u}^{e s t}(\boldsymbol{x})\right\rangle_{Y}=\boldsymbol{u}^{d} & \forall \boldsymbol{x} \in \boldsymbol{\Gamma}_{\boldsymbol{u}} \\ \left\langle\boldsymbol{\sigma}^{e s t}(\boldsymbol{x})\right\rangle_{Y} \cdot \boldsymbol{n}=\boldsymbol{F}^{d} & \forall \boldsymbol{x} \in \boldsymbol{\Gamma}_{\boldsymbol{t}}\end{cases}
$$

with the macroscale stress: $\left\langle\boldsymbol{\sigma}^{e s t}\right\rangle_{Y}=\boldsymbol{\Sigma}$ and strain: $\left\langle\varepsilon^{e s t}\right\rangle_{Y}=\boldsymbol{E}$.

It is apparent from problem (27d) that the boundary condition on $\boldsymbol{\Gamma}_{\boldsymbol{t}}$ is only satisfied by the mean value of $\boldsymbol{\sigma}^{\text {est }}$, therefore:

$$
\boldsymbol{\sigma}^{e s t} \cdot \boldsymbol{n} \neq \boldsymbol{F}^{d}
$$

For this reason, added to the fact that the local stress field is generally not periodic in the vicinity of the Neumann boundaries, $\boldsymbol{\sigma}^{\text {est }}$ is inaccurate near the boundaries. To overcome this, it was proposed in Ref. [35] to correct the expansion of the displacement and the associated expansion of the stresses in the neighborhood of the boundary of a 2D problem. Boundary layers term $\boldsymbol{\sigma}^{b l}$ are introduced whose sum with local stresses satisfies exactly boundary conditions at each microscopic point. We propose to compute $\boldsymbol{\sigma}^{b l}$ by considering six auxiliary problems on the unit-cell subjected to six characteristic loads $F_{k l}$ with $k l=\{11,22,33,23,31,12\}$ on the 
surface where the Neumann boundary condition is applied. The opposite surface is fixed, and other surfaces of the unit-cell are subjected to periodicity conditions. The characteristic load is defined as follows:

$$
F_{i k l}=-B_{i j k l} \cdot n_{j}+\frac{1}{|Y|} \int_{Y} B_{i j k l} \cdot n_{j} d Y \quad \text { with fixed index } j
$$

where $n_{j}$ is the normal direction of $\boldsymbol{\Gamma}_{\boldsymbol{t}}$ and the fixed index $j \in\{1,2,3\}$ is the index of this normal direction. $B_{i n k l}$ are the components of the stress relocalization tensor $\mathbb{B}$.

The resulting displacements, strains, and stresses solving each auxiliary problem are the components of the boundary-layer relocalization tensors $\mathbb{D}^{b l}, \mathbb{A}^{b l}$, and $\mathbb{B}^{b l}$, respectively. Thus, the boundary layer corrective terms are computed as follows:

$$
\left\{\begin{array}{l}
\boldsymbol{\sigma}^{b l}=\mathbb{B}^{b l}: \boldsymbol{E} \\
\boldsymbol{\varepsilon}^{b l}=\mathbb{A}^{b l}: \boldsymbol{E} \\
\boldsymbol{u}^{b l}=\mathbb{D}^{b l}: \boldsymbol{E}
\end{array}\right.
$$

As a result, the stress field $\boldsymbol{\sigma}^{\text {cor }}$

$$
\boldsymbol{\sigma}^{c o r}=\boldsymbol{\sigma}^{e s t}+\boldsymbol{\sigma}^{b l}
$$

satisfy the Neumann BC.

\subsection{Correctors for Dirichlet BCs}

We recall that the proposed estimated displacement field is given by equation (26a):

$$
\boldsymbol{u}^{e s t}(\boldsymbol{x})=\boldsymbol{U}(\boldsymbol{x})+\boldsymbol{u}^{1}(\boldsymbol{x})
$$

with $\boldsymbol{u}^{1}=\mathbb{D}: \boldsymbol{E}$ is the first-order corrector of the displacement field obtained from the resolution of $\left(\mathcal{P}_{\text {order }}^{1^{\text {st }}}\right)$. The displacement $\boldsymbol{u}^{\text {est }}$ estimates correctly the real displacement in the interior of the material where the structure is periodic. Nevertheless, we need to ensure that $\boldsymbol{u}^{\text {est }}$ verifies the Dirichlet boundary condition $\boldsymbol{u}^{\text {est }}=\boldsymbol{u}^{d}$ on $\boldsymbol{\Gamma}_{\boldsymbol{u}}$. But since $\boldsymbol{U}=\boldsymbol{u}^{d}$ on $\boldsymbol{\Gamma}_{\boldsymbol{u}}$ is enforced then $\boldsymbol{u}^{\text {est }}$ do not necessarily verify the Dirichlet boundary conditions.

Therefore, a correction is needed that is the negative of $\boldsymbol{u}^{1}$ at the boundary and decays inward, and such that the corrected field would read:

$$
\boldsymbol{u}^{\text {cor }}(\boldsymbol{x})=\boldsymbol{U}(\boldsymbol{x})+\left(\boldsymbol{u}^{1}(\boldsymbol{x})+\boldsymbol{u}^{b l}(\boldsymbol{x})\right)
$$


The correction $\boldsymbol{u}^{b l}$ must verify:

$$
\boldsymbol{u}^{b l}(\boldsymbol{x})=-\boldsymbol{u}^{1}(\boldsymbol{x}) \quad \forall \boldsymbol{x} \in \boldsymbol{\Gamma}_{\boldsymbol{u}}
$$

As in the Neumann case, an auxiliary problem is considered on which the boundary layer fields will be computed. In this case, corrective displacements $\varrho_{k l}$ with $k l=\{11,22,33,23,31,12\}$ are applied to the unit-cell and defined as:

$$
\varrho_{i k l}=-D_{i k l}
$$

where $D_{i j k}$ are the components of the displacement relocalization tensor $\mathbb{D}$. The corrective boundary layer fields are obtained by conducting the same analyses defined in Eq. (30),(31), and (32).

\subsection{Mixed $B C s$}

The correction for boundary layers with mixed BCs is conducted by considering six auxiliary problems on the unit-cell subjected to both six characteristic loads $F_{i k l}$ defined in Eq. (29) and displacements $\boldsymbol{\varrho}_{i k l}$ defined in Eq. (37), with $k l=\{11,22,33,23,31,12\}$. The correction to be ultimately applied for each $i$ index depends on the actual (Neumann or Dirichlet) BC applied in this specific direction. For the purpose of illustration, we shall consider the following simple of a composite in tension (see Fig. 4):

\subsection{Proposed general boundary correction scheme}

The proposed boundary correction strategy is summarized in Fig. 6. For the sake of simplicity and clarity, an invariance along the thickness direction is considered. The structure in Fig. 6 is fixed on $\Gamma_{u}$, a surface traction is applied on $\Gamma_{t}$ and other boundaries of $\Gamma_{t}$ are kept free of forces. The estimated fields are correct in the bulk region where the structure is periodic and the boundary layers to be corrected are divided into five regions. For what follows, $k l=\{11,22,33,23,31,12\}$ and $i \in\{1,2,3\}$. The five regions are:

1. Boundary layers subjected to Dirichlet BCs:

The unit-cell is subjected to six displacement vectors $\varrho_{i k l}(37)$ on the face where Dirichlet BCs are applied, the opposite face is kept free of forces. Remaining faces of the unit-cell are subjected to periodicity conditions (see Fig. 5a).

2. Corner cells subjected to both Neumann and Dirichlet BCs:

The unit-cell, which is a corner cell in this case as shown Fig. 6, is subjected to six displacement vectors $\varrho_{i k l}(37)$ on the face where Dirichlet BCs are applied and six loads $\boldsymbol{F}_{i k l}$ (29) on the face where Neumann BCs are applied. Remaining faces of the unit-cell are kept free of forces (see Fig. 5b). 


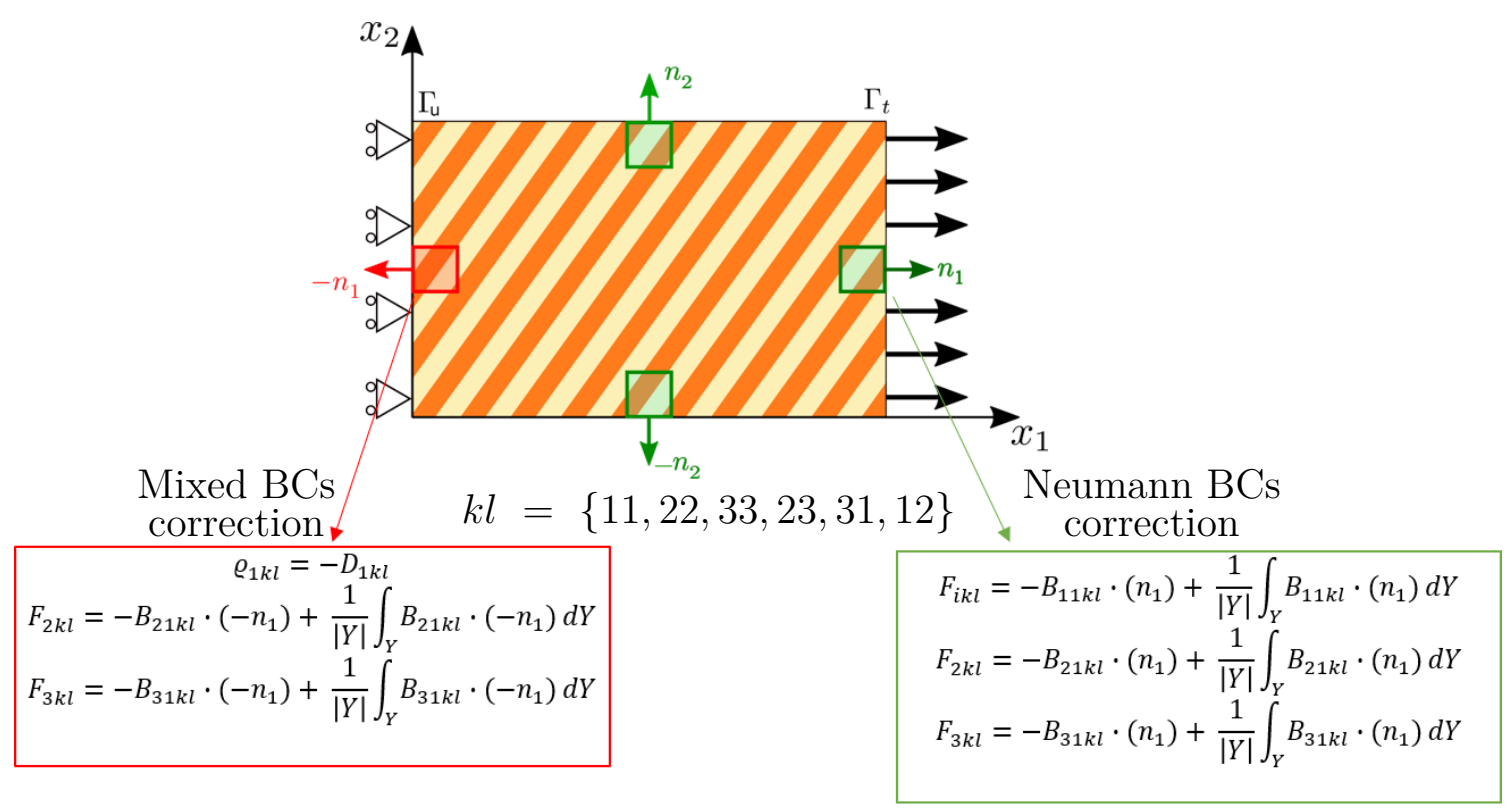

Figure 4: Illustration of the Neumann and mixed BCs corrections to be applied to the unit-cell.

3. Free forces boundary layers:

The unit-cell is subjected to six loads $\boldsymbol{F}_{i k l}(29)$ where the unit-cell is free of forces. The opposite face where the loads are applied is fixed. Remaining faces of the unit-cell are subjected to periodicity conditions (see Fig. 5c).

4. Boundary layers subjected to Neumann BCs:

The unit-cell is subjected to six loads $\boldsymbol{F}_{i k l}(29)$ on the face where Neumann BCs are applied. The opposite face where the loads are applied is fixed. Remaining faces of the unit-cell are subjected to periodicity conditions (see Fig. 5d).

5. Corner cells subjected to Neumann BCs:

The unit-cell is subjected to six loads $\boldsymbol{F}_{i k l}(29)$ where Neumann BCs are applied and where the unit-cell is free of forces. Remaining faces of the unit-cell are fixed (see Fig. 5e).

The boundary layer correction and relocalization tensors $\mathbb{D}^{b l}, \mathbb{A}^{b l}$, and $\mathbb{B}^{b l}$ are constructed from the resolution of auxiliary problems. Thereafter corrective boundary layer fields are computed and added to estimated fields as summarized in Fig. 6. 


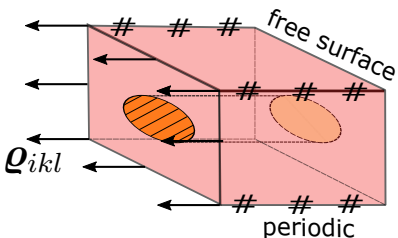

(a) Correction for a cell subjected to Dirichlet $\mathrm{BCs}$

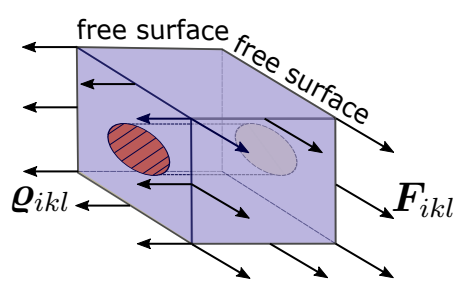

(b) Correction for a corner subjected to Neumann \& Dirichlet BCs

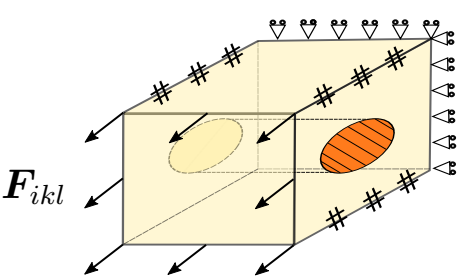

(c) Correction for a free cell

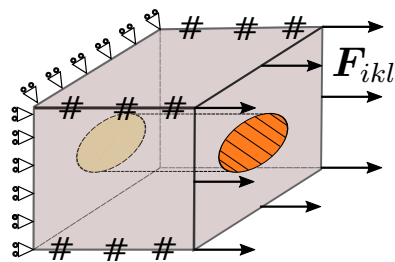

(d) Correction for a cell subjected to Neumann BCs

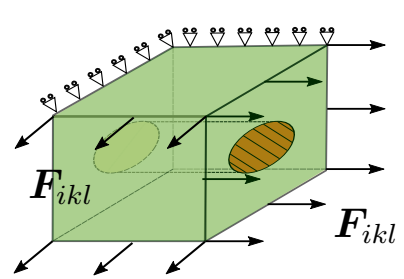

(e) Correction for a corner subjected to Neumann BCs

Figure 5: Illustration of auxiliary problems for boundary layers correction. 


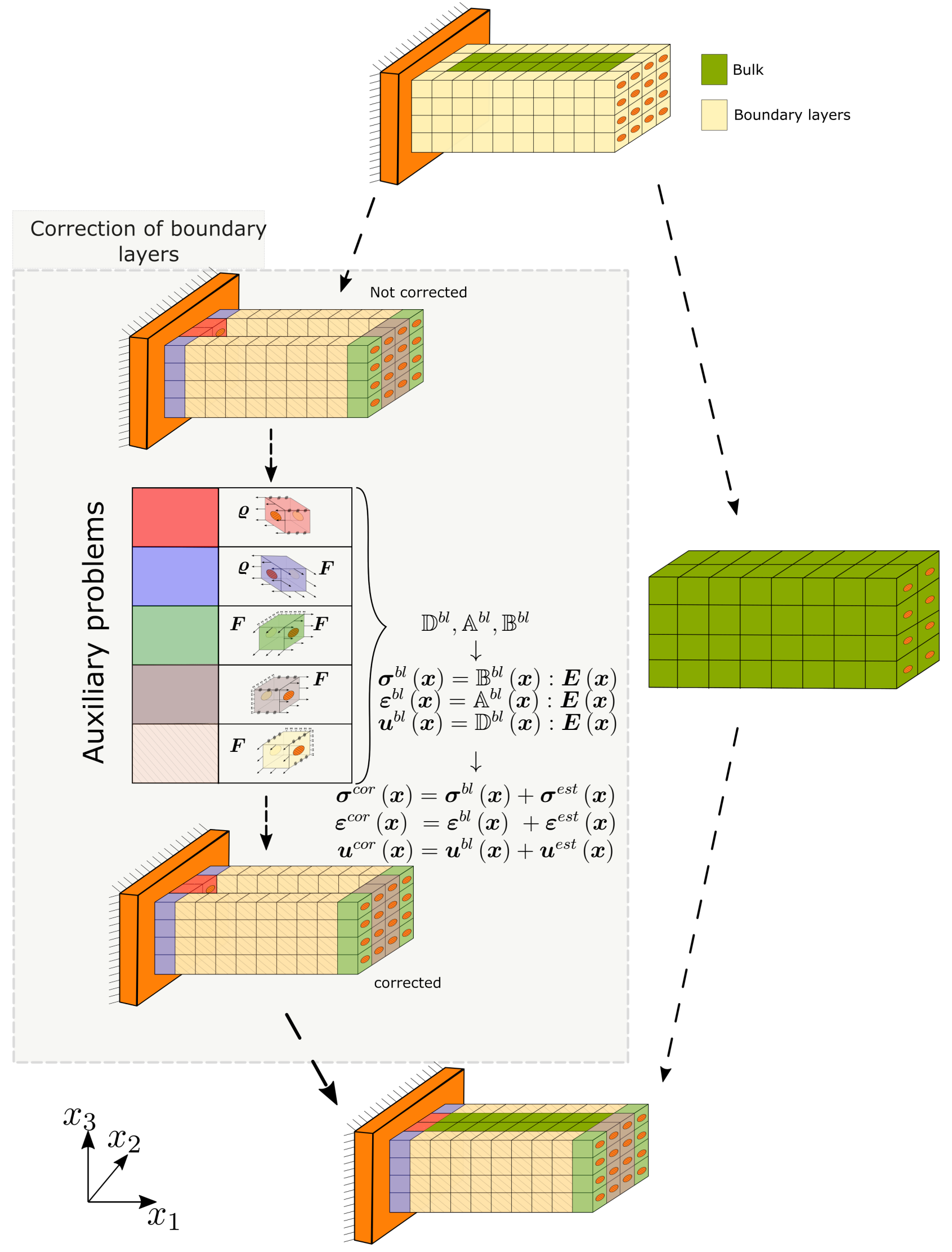

Figure 6: Illustration of the boundary layer correction process. An invariance along the vertical (thickness) direction is considered for simplicity. 


\section{Numerical examples}

In this section, numerical examples are presented and discussed in order to demonstrate the efficiency and limitations of the suggested approach. For illustrative purposes, a simple study conducted on a uni-directional laminate in tension is presented in appendix $\mathrm{B}$.

\subsection{Matrix-inclusion composite}

We consider the plane strain linear elasticity problem of a matrix-inclusion composite in tension as depicted in Fig. 7. The size of the structure is $L=8 \mathrm{~mm}, H=$ $5 \mathrm{~mm}$ and $W=1 \mathrm{~mm}$, and the diameter of the fibers is $D=0.2 \mathrm{~mm}$. The matrix and the spherical inclusions are assumed to be isotropic linear elastic with coefficients $\left(E_{m}=1000 \mathrm{MPa}, \nu_{m}=0.3\right)$ and $\left(E_{f}, \nu_{f}=0.3\right)$ where $E_{f}$ is varied in the examples, respectively. We propose to estimate the microscale fields by using the relocalization

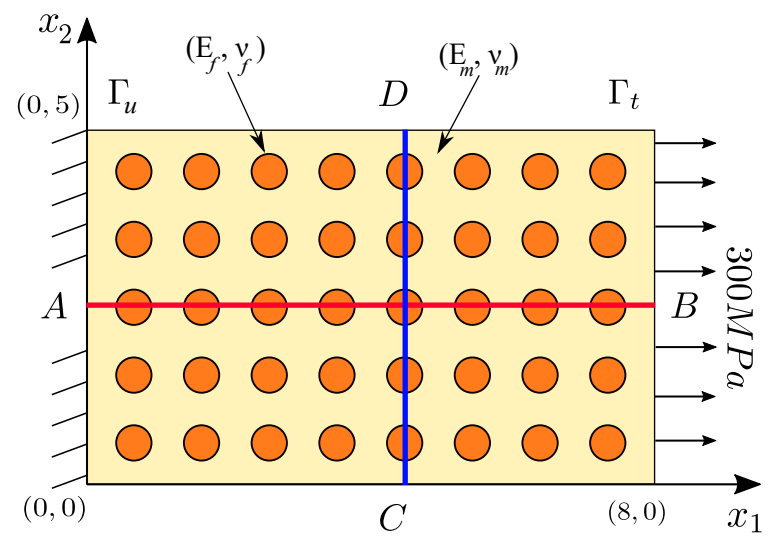

(a) Structure geometry

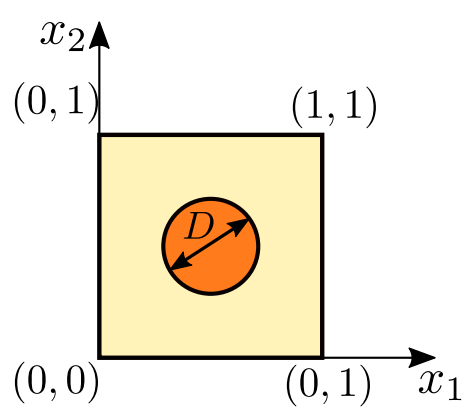

(b) unit-cell domain

Figure 7: Illustration of matrix-inclusion composite. The structure is fixed on $\Gamma_{u}$ and a non-zero surface traction is applied on $\Gamma_{t}$, other boundaries are kept free. The results will be plotted along $\mathrm{AB}$ and $\mathrm{CD}$ lines.

procedure described in subsection. 2.3, and correct these estimates on the boundaries based on the proposed boundary correction scheme described in Sec. 3.

In this study we will compare the ensuing stress fields:

- The microscale stress field obtained by solving the problem $\left(\mathcal{P}_{\text {ref }}\right)$ using DNS, which will be considered as our reference, indexed ref.

- The homogeneous stress field obtained from solving the problem $\left(\mathcal{P}_{\text {hom }}\right)$, indexed hom. 
- The proposed estimate of the microscale stress field obtained by the relocalization process, indexed est.

- The correction of the microscale stress field corrected with the proposed boundary layer correction scheme, indexed cor.

We will also quantify the difference between the reference and estimated fields by computing the modeling error. For this purpose, we define the following local (element wise) error in energy norm:

$$
\begin{aligned}
\|\boldsymbol{e}\|_{E\left(\Omega_{e}\right)} & =\left\|\boldsymbol{u}^{r e f}(\boldsymbol{x})-\boldsymbol{u}^{k}(\boldsymbol{x})\right\|_{E\left(\Omega_{e}\right)} \\
& =\left(\int_{\Omega_{e}} \nabla^{s}\left(\boldsymbol{u}^{r e f}(\boldsymbol{x})-\boldsymbol{u}^{k}(\boldsymbol{x})\right): \mathbb{C}: \nabla^{s}\left(\boldsymbol{u}^{r e f}(\boldsymbol{x})-\boldsymbol{u}^{k}(\boldsymbol{x})\right) d \Omega_{e}\right)^{\frac{1}{2}}
\end{aligned}
$$

where $\Omega_{e}$ denotes the domain of an element and $\boldsymbol{u}^{k}(\boldsymbol{x})$ denotes the estimated displacement field whose error is measured $(k=e s t$ or $k=c o r)$. The global error $\|\boldsymbol{e}\|_{E(\Omega)}$ is then defined as:

$$
\|\boldsymbol{e}\|_{E(\Omega)}^{2}=\sum_{e}\|\boldsymbol{e}\|_{E\left(\Omega_{e}\right)}^{2}
$$

\subsubsection{Comparison of stress fields}

We consider a contrast $\frac{E_{f}}{E_{m}}=10$, where $E_{f}$ and $E_{m}$ are Young's moduli of the fiber and matrix phases, respectively. Comparison of resulting stress fields along $\mathrm{AB}$

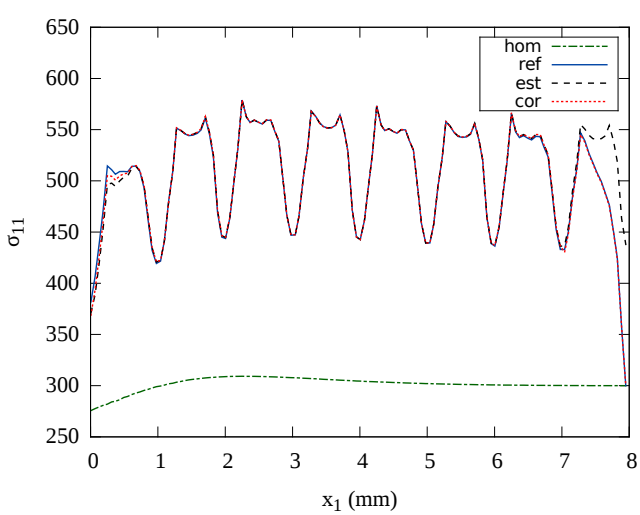

(a) $\sigma_{11}(\mathrm{MPa})$

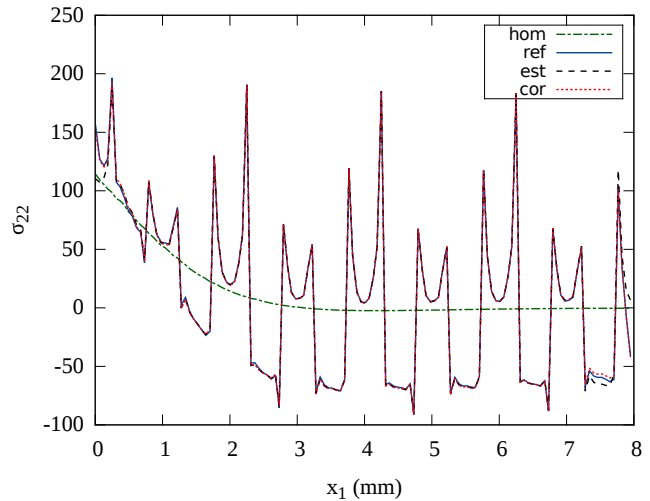

(b) $\sigma_{22}(\mathrm{MPa})$

Figure 8: Results of the homogenized (hom), reference (ref), estimated (est) and corrected (cor) stress fields plotted along the AB line with $\frac{E_{f}}{E_{m}}=10$ for the matrix-inclusion composite in tension. 
and CD lines are presented in Fig. 8 and Fig. 9, respectively. The corresponding $\mathrm{AB}$ and $\mathrm{CD}$ lines are defined on Fig. 7. Both $\sigma_{11}^{\text {est }}$ and $\sigma_{22}^{\text {est }}$ perfectly coincide with reference ones in the inner domain of the composite, where they are periodic. Nevertheless accuracy is lost near the boundaries. After the boundary layer correction, the corrected fields provide a good approximation of the reference near the boundaries. In particular, $\sigma_{11}^{\text {cor }}$ verifies the applied Neumann condition $(300 \mathrm{MPa})$ at $x_{1}=8 \mathrm{~mm}$ as for the reference and the homogeneous counterparts, but it is not the case for $\sigma_{11}^{e s t}$. More precise quantification of the error is provided in the next subsection.

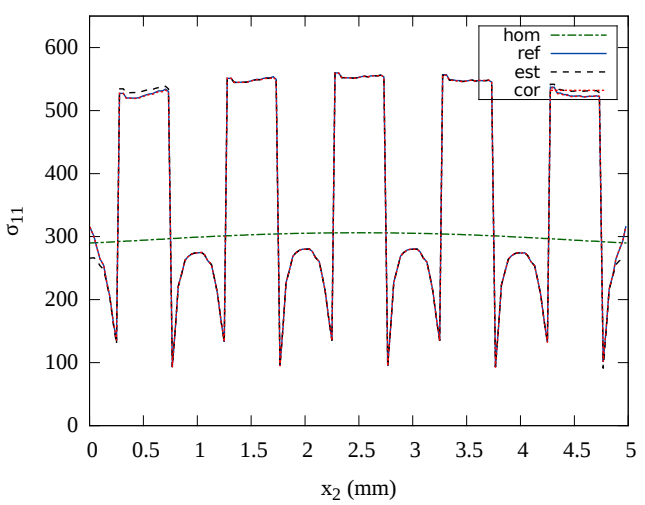

(a) $\sigma_{11}(\mathrm{MPa})$

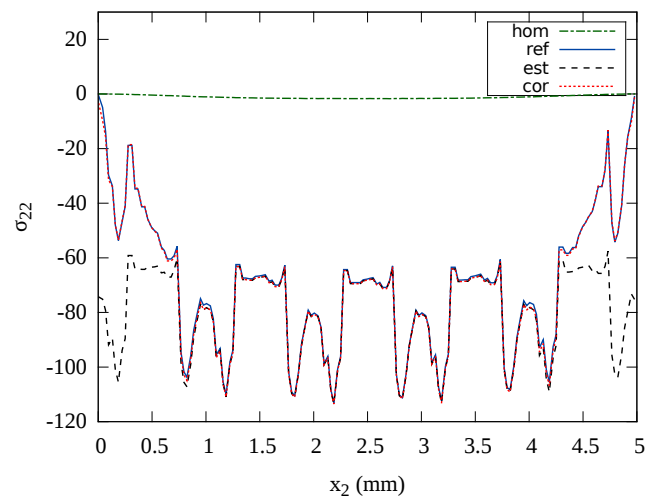

(b) $\sigma_{22}(\mathrm{MPa})$

Figure 9: Results of the homogenized (hom), reference (ref), estimated (est) and corrected (cor) stress fields plotted along the CD line with $\frac{E_{f}}{E_{m}}=10$ for the matrix-inclusion composite in tension.

\subsubsection{Modeling error}

The local relative modeling error without the boundary layer correction, presented in Fig. 10a, is concentrated near the boundaries and maximal in the vicinity of the applied Neumann boundary. This is mainly explained by the loss of periodicity in the vicinity of the boundaries. After the correction, the error is significantly reduced near the boundaries. We notice that the remaining error is mainly concentrated at the corners, especially near the Neumann boundary. We recall that a particular treatment was considered for the corners correction, as explained in Fig. 6. This correction improves the overall result but seems to be still imperfect since errors remain at the interfaces. Table 1 summarizes the global relative modeling error for different contrast ratios. The boundary layer correction allows to drastically reduce the modeling error by a factor of 4 to 5 for the four ratios. This demonstrates that 


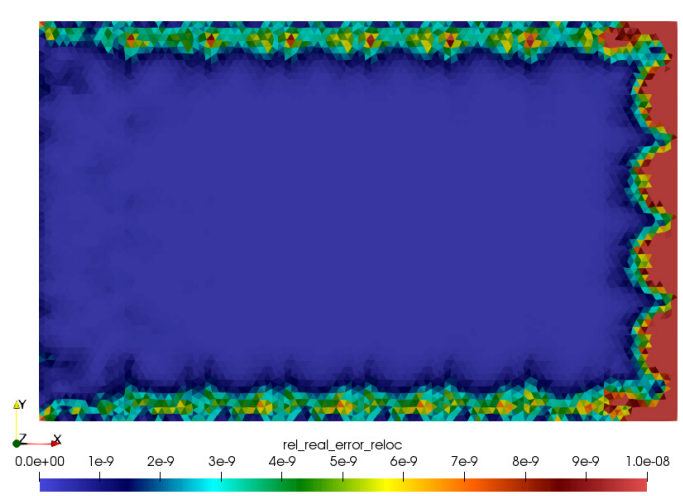

(a) $\frac{\left\|\boldsymbol{u}^{r e f}-\boldsymbol{u}^{e s t}\right\|_{E\left(\Omega_{e}\right)}^{2}}{\left\|\boldsymbol{u}^{r e f}\right\|_{E(\Omega)}^{2}}$

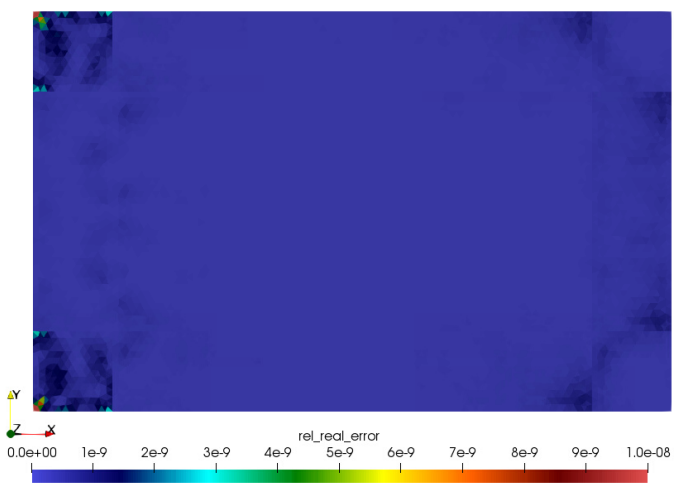

(b) $\frac{\left\|\boldsymbol{u}^{r e f}-\boldsymbol{u}^{\text {cor }}\right\|_{E\left(\Omega_{e}\right)}^{2}}{\left\|\boldsymbol{u}^{r e f}\right\|_{E(\Omega)}^{2}}$

Figure 10: Comparison of the local relative modeling error results with $\frac{E_{f}}{E_{m}}=10$ for the matrixinclusion composite in tension.

the correction process is effective even for a higher contrast between fiber and matrix phases.

Table 1: Comparison of the global relative modeling error for different contrast ratios for the matrixinclusion composite in tension.

\begin{tabular}{ccccc}
\hline $\begin{array}{c}\text { Global modeling } \\
\text { error }\end{array}$ & $\begin{array}{c}\text { Contrast } \\
10\end{array}$ & $\begin{array}{c}\text { Contrast } \\
25\end{array}$ & $\begin{array}{c}\text { Contrast } \\
50\end{array}$ & $\begin{array}{c}\text { Contrast } \\
100\end{array}$ \\
\hline$\frac{\left\|\boldsymbol{u}^{\text {ref }}-\boldsymbol{u}^{\text {est }}\right\|_{E(\Omega)}}{\left\|\boldsymbol{u}^{\text {ref }}\right\|_{E(\Omega)}}$ & 0.038 & 0.044 & 0.046 & 0.048 \\
$\frac{\left\|\boldsymbol{u}^{\text {ref }}-\boldsymbol{u}^{\text {cor }}\right\|_{E(\Omega)}}{\left\|\boldsymbol{u}^{\text {ref }}\right\|_{E(\Omega)}}$ & 0.009 & 0.010 & 0.011 & 0.012
\end{tabular}

\subsection{Laminated composite in tension}

We consider a plane strain elasticity problem of a laminated composite made of two layers as presented in Fig. 11. The size of the structure is $L=8 \mathrm{~mm}, H=5 \mathrm{~mm}$ and $W=1 \mathrm{~mm}$. The two layers are assumed to be isotropic linear elastic with coefficients $\left(E_{m}=1000 \mathrm{MPa}, \nu_{m}=0.3\right)$ and $\left(E_{f}, \nu_{f}=0.3\right)$ where $E_{f}$ is varied in the examples. We conduct the same study as in the previous example. In order to correct the $\Gamma_{u}$ boundary layer, it is necessary to apply both the Neumann and Dirichlet boundary corrections. 


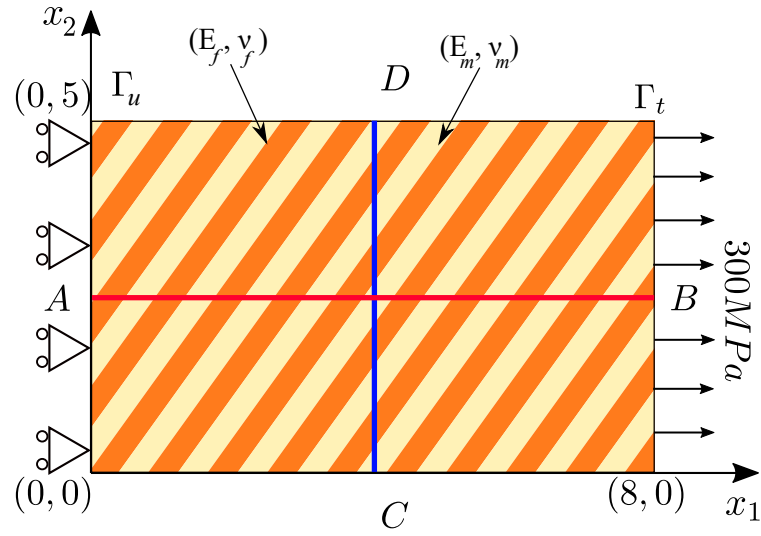

(a) Structure geometry

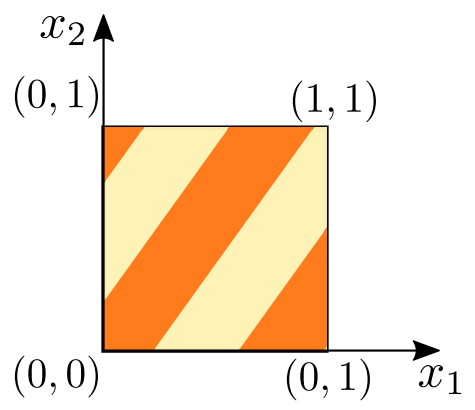

(b) unit-cell domain

Figure 11: Illustration of the laminated composite in tension. The structure is sliding on $\Gamma_{u}$ and a surface tension is applied on $\Gamma_{t}$. Other boundaries are kept free. Results will be plotted along AB and CD lines.

\subsubsection{Comparison of stress fields}

Figs. 12 and 13 show that $\sigma^{\text {cor }}$ is in agreement with $\sigma^{\text {ref }}$ in the inner domain of the composite and on the boundaries. We also notice that high stresses are developed in the vicinity of the sliding boundary $\Gamma_{u}$, especially for higher contrast ratios. For a ratio of 50 , the estimated stress is 3 times smaller than the real stress for $\sigma_{11}$ and 7 times smaller for $\sigma_{12}$, which may result in underestimating failure criteria if the design is conducted without any boundary layer correction.

\subsubsection{Modeling error}

The local relative modeling error between the reference and estimated fields (Fig. 14a) is negligible in the inner domain of the composite but significant on the boundaries, especially in the vicinity of the sliding boundary $\Gamma_{u}$. After the correction (Fig. 14b), the modeling error is significantly reduced, and remains concentrated at the corners of $\Gamma_{u}$.

Table 2 summarizes the relative modeling error for different contrast ratios. Similarly, by correcting the boundary layers, the remaining error is divided by 3 for the four ratios.

\subsection{Laminated composite in bending}

The same composite as in the previous example is now subjected to bending as shown in Fig. 15. This specific load will create high macroscale strain gradients. In this case, the first-order approximation is expected to lose its accuracy even in the 


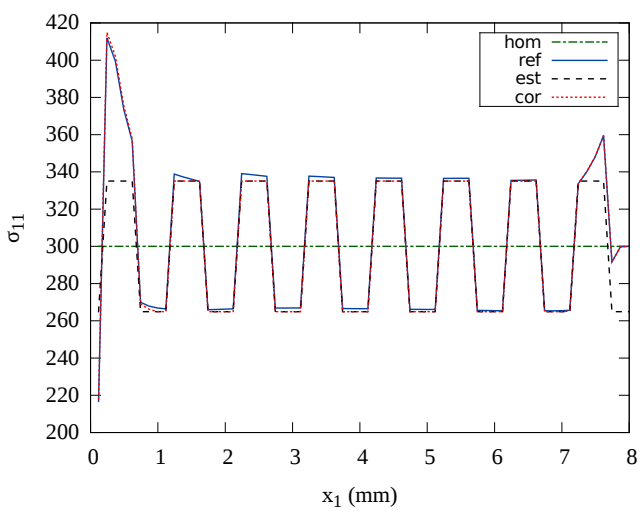

(a) $\sigma_{11}(\mathrm{MPa})$

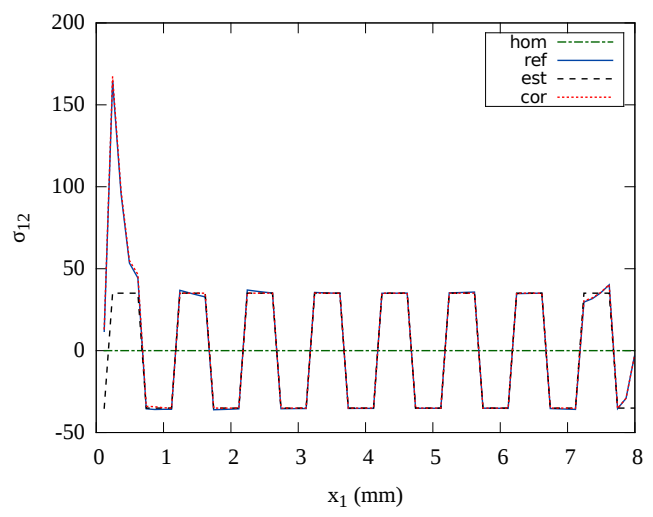

(b) $\sigma_{12}(\mathrm{MPa})$

Figure 12: Results of the homogenized (hom), reference (ref), estimated (est) and corrected (cor) stress fields plotted along the $\mathrm{AB}$ line with $\frac{E_{f}}{E_{m}}=10$ for the laminated composite in tension.

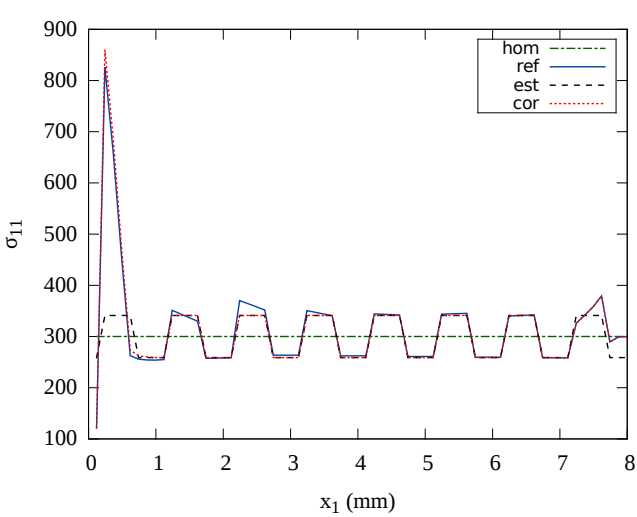

(a) $\sigma_{11}(\mathrm{MPa})$

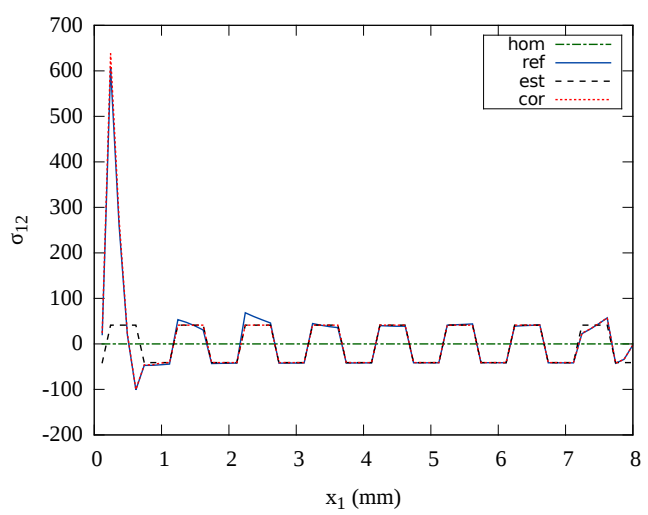

(b) $\sigma_{12}(\mathrm{MPa})$

Figure 13: Results of the homogenized (hom), reference (ref), estimated (est) and corrected (cor) stress fields plotted along the $\mathrm{AB}$ line with $\frac{E_{f}}{E_{m}}=50$ for the laminated composite in tension.

inner domain of the structure. In order to illustrate this, the same study is conducted by comparing stress fields and computing the modeling error.

\subsubsection{Comparison of stress fields}

We only consider a contrast of 10 in this example, as it will be sufficient to illustrate our point. Comparisons of the stress fields along the AB line are presented 

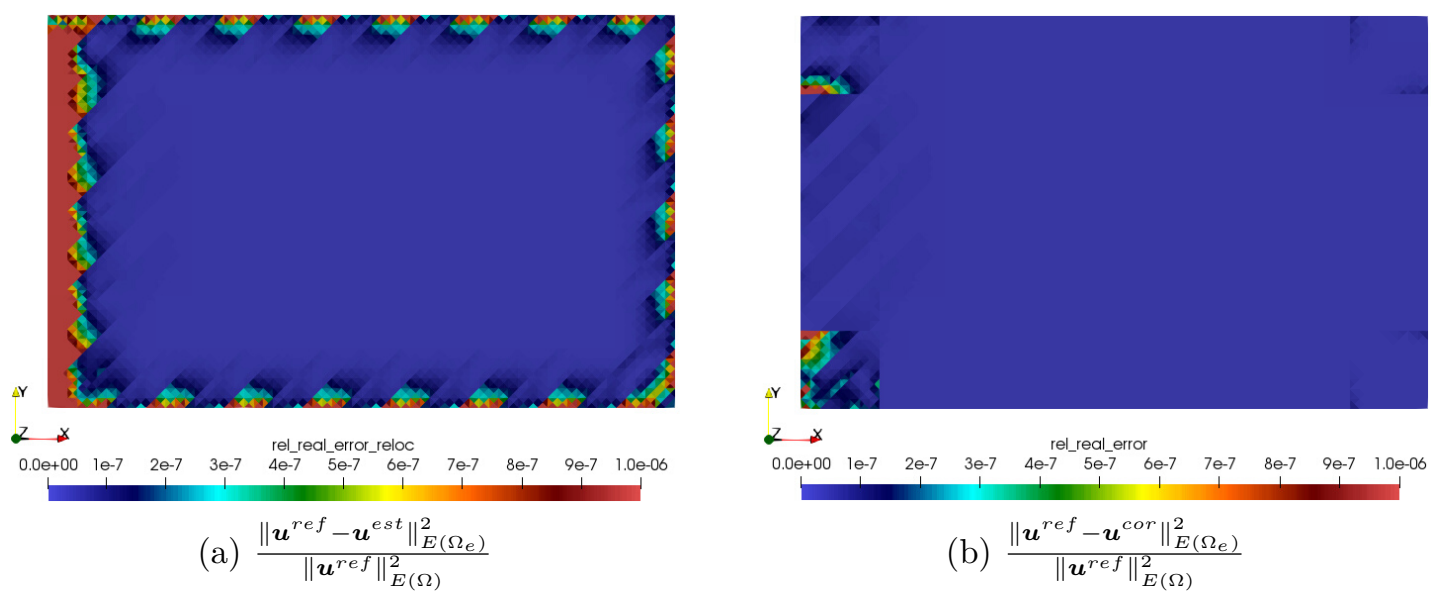

Figure 14: Comparison of the local relative modeling error results for $\frac{E_{f}}{E_{m}}=10$ for the laminated composite in tension.

Table 2: Comparison of the global relative modeling error for different contrast ratios for the laminated composite in tension.

\begin{tabular}{ccccc}
\hline $\begin{array}{c}\text { Global modeling } \\
\text { error }\end{array}$ & $\begin{array}{c}\text { Contrast } \\
10\end{array}$ & $\begin{array}{c}\text { Contrast } \\
25\end{array}$ & $\begin{array}{c}\text { Contrast } \\
50\end{array}$ & $\begin{array}{c}\text { Contrast } \\
100\end{array}$ \\
\hline$\frac{\left\|\boldsymbol{u}^{\text {ref }}-\boldsymbol{u}^{e s t}\right\|_{E(\Omega)}}{\left\|\boldsymbol{u}^{\text {ref }}\right\|_{E(\Omega)}}$ & 0.10 & 0.14 & 0.17 & 0.20 \\
$\frac{\left\|\boldsymbol{u}^{\text {ref }}-\boldsymbol{u}^{\text {cor }}\right\|_{E(\Omega)}}{\left\|\boldsymbol{u}^{\text {ref }}\right\|_{E(\Omega)}}$ & 0.027 & 0.036 & 0.047 & 0.066
\end{tabular}

in Fig. 16 and along the CD line in Fig. 17.

The results show that the estimated fields, contrary to the previous examples, are inaccurate even in the inner domain of the structure. This is mainly explained by high macroscale strain gradients induced by the bending. To overcome this shortcoming, higher-order terms must be introduced in the asymptotic expansion which features higher-order gradients of macroscale strain and characteristic tensors of the microstructure [16]. Despite this clear limitation, the corrected estimated stresses are still a better approximation of the real stresses near the boundaries.

\subsubsection{Modeling error}

The local relative modeling error before the correction is maximal in the vicinity of the sliding boundary $\Gamma_{u}$ but also spread across the structure as shown in Fig. 18a. 


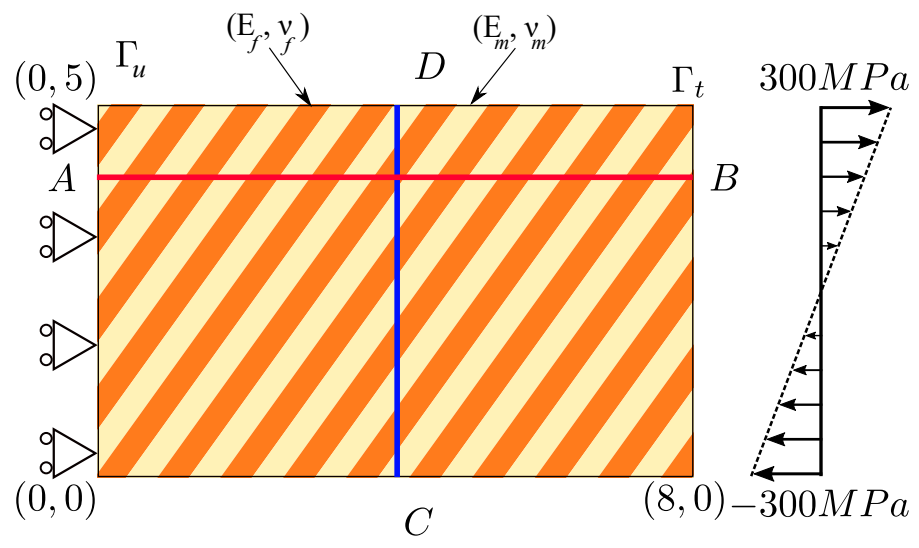

Figure 15: Structure composed of a laminated composite subjected to bending. Results will be plotted along $\mathrm{AB}$ and $\mathrm{CD}$ lines.

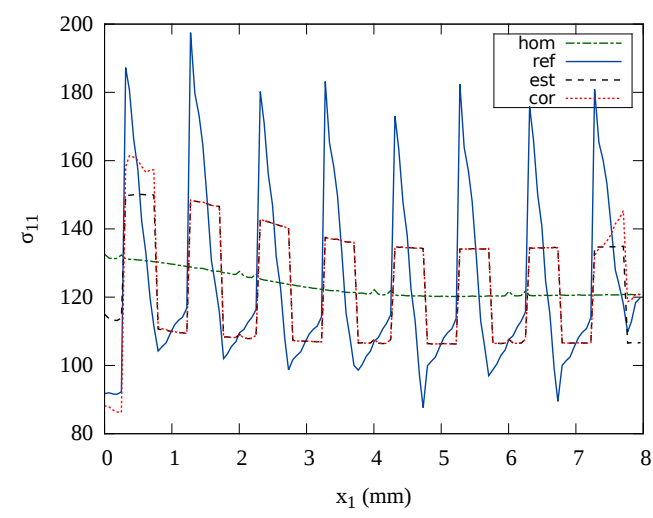

(a) $\sigma_{11}(\mathrm{MPa})$

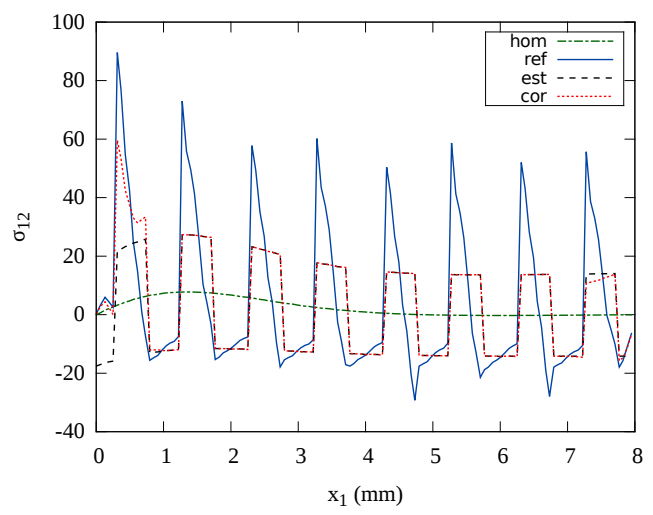

(b) $\sigma_{12}(\mathrm{MPa})$

Figure 16: Results of the homogenized (hom), reference (ref), estimated (est) and corrected (cor) stress fields plotted along the $\mathrm{AB}$ line with $\frac{E_{f}}{E_{m}}=10$ for the laminated composite in bending.

After the boundary layer correction, the error is considerably reduced on the boundaries but still present inside the structure as shown in Fig. 18b.

\section{Conclusions}

In this study, we propose a general boundary layer correction based on asymptotic homogenization in order to estimate consistent microscale fields in the vicinity of the boundaries. The main idea of the method is to compute corrective terms from solving different auxiliary problems on the unit-cell. The nature of the problem to 


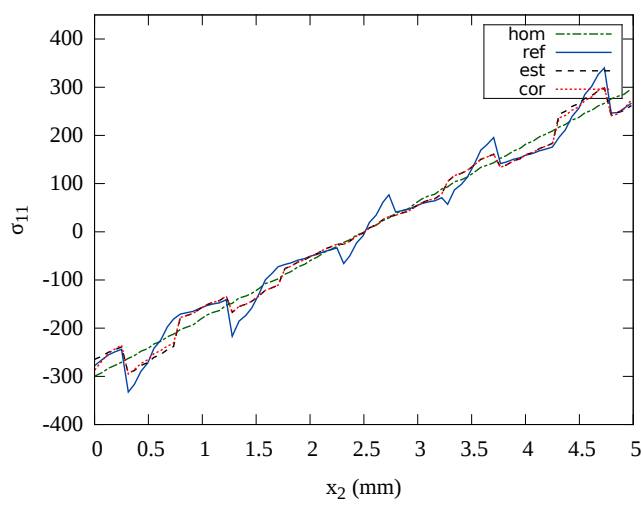

(a) $\sigma_{11}(\mathrm{MPa})$

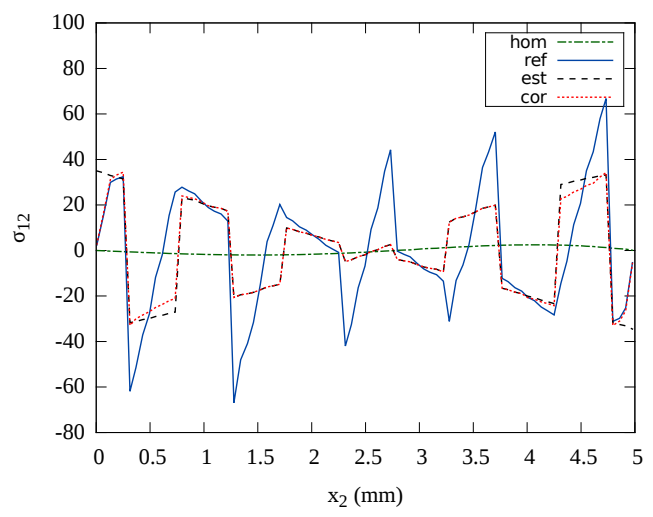

(b) $\sigma_{12}(\mathrm{MPa})$

Figure 17: Results of the homogenized (hom), reference (ref), estimated (est) and corrected (cor) stress fields plotted along the CD line with $\frac{E_{f}}{E_{m}}=10$ for the laminated composite in bending.

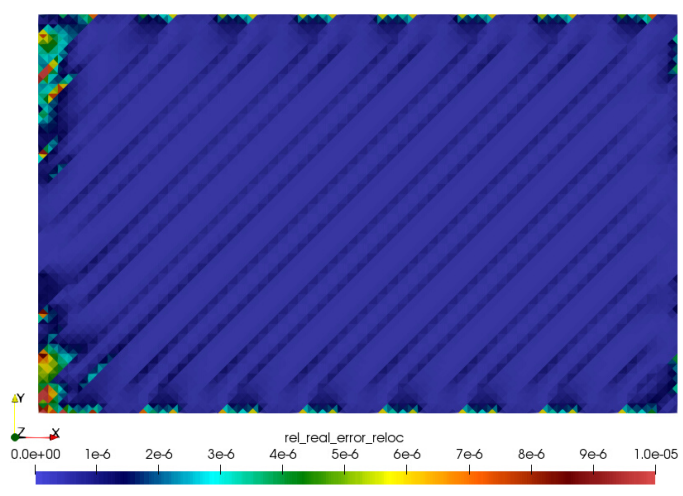

(a) $\frac{\left\|\boldsymbol{u}^{r e f}-\boldsymbol{u}^{e s t}\right\|_{E\left(\Omega_{e}\right)}^{2}}{\left\|\boldsymbol{u}^{r e f}\right\|_{E(\Omega)}^{2}}$

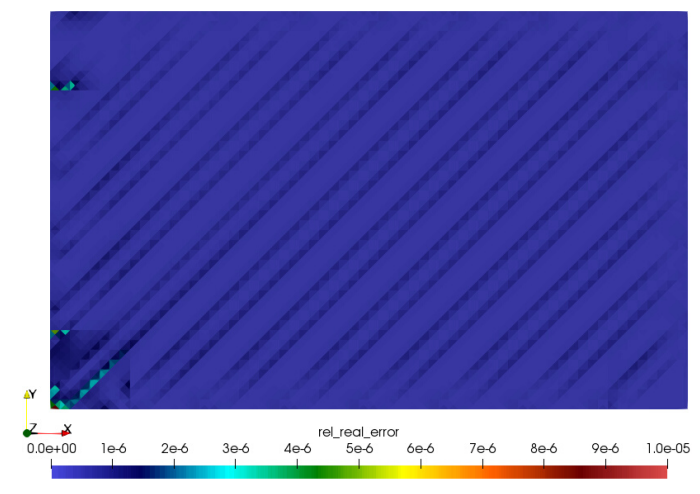

(b) $\frac{\left\|\boldsymbol{u}^{r e f}-\boldsymbol{u}^{c o r}\right\|_{E\left(\Omega_{e}\right)}^{2}}{\left\|\boldsymbol{u}^{r e f}\right\|_{E(\Omega)}^{2}}$

Figure 18: Comparison of the local relative modeling error results for $\frac{E_{f}}{E_{m}}=10$ for the laminated composite in bending.

solve depends on the local boundary conditions. These corrective terms are added to the usual fields obtained from the relocalization process, drastically improving the solution near the boundaries.

It was shown, by means of three numerical examples, that the corrected fields successfully predict stress or strain concentrations near the boundaries, which can be responsible for the failure of the individual phases. The proposed method was developed for different boundary conditions (Dirichlet, Neumann, and mixed). The 
associated computational cost is relatively inexpensive since the corrective terms are computed over the unit-cell. All localization tensors can be computed off-line and used for any composite structure involving the same unit-cell.

The major conclusions that can be drawn from this study are:

- The first-order estimated fields, obtained from the relocalization process, are a good approximation of the heterogeneous (reference) fields in the inner domain of the structure. This result is not true for cases when high gradients of macroscale strains exist.

- The boundary layer correction allows to reduce the global modeling error by a factor of 3 to 4 for different ratios $\frac{E_{f}}{E_{m}}$.

- The corrected stress field captures high stresses developed near the boundary region. Particularly, for laminated composite in traction (ratio of $\frac{E_{f}}{E_{m}}=50$ ) shown in Sec. 4.2, the estimated stress is 3 times smaller than the real stress for $\sigma_{11}$ and 7 times smaller for $\sigma_{12}$. This may result in underestimating failure criteria if the design is conducted without any boundary layer correction.

The method can now be applied with confidence to more realistic engineering composite structures, especially in the 3D case for which DNS are not reasonably possible. This also implies the use of coarse macroscale finite element meshes and proper projection/interpolation to the unit-cell mesh.

The limitation of the proposed approach have also been evidenced in some cases on which it has been shown that boundary conditions are not the only source of modeling error. Second-gradient effects, which first-order asymptotic homogenization neglects, may play a prominent role. Combining higher-order and boundary correctors could be a path toward efficient error estimation or adaptive model refinement.

\section{Declaration of competing interest}

The authors declare that they have no known competing financial interests or personal relationships that could have appeared to influence the work reported in this paper.

\section{Acknowledgments}

This work was supported by the French National Association for Research and Technology (ANRT) through the research grant CIFRE 2019/1715. 


\section{Appendix A: Finite element resolution}

This appendix describes the numerical procedure using the Finite Element Method $(\mathrm{FEM})$ in order to solve $\left(\mathcal{P}_{\text {order }}^{1^{\text {st }}}\right)$ and thus compute the relocalization tensors $(\mathbb{D}, \mathbb{A}, \mathbb{B})$ and the homogenized elasticity tensor $\mathbb{C}^{0}$. We also describe the proposed numerical relocalization scheme used to estimate the full microscopic fields.

\section{Computation of relocalization and homogenized elasticity matrices}

The displacement corrector $\boldsymbol{u}^{1}$, solution of the first-order periodic problem, constitute the components of the corrector displacement matrix [DD]. The finite element approximation to Eq. (14) is:

$$
\begin{aligned}
\int_{Y^{e}}[\boldsymbol{B}]_{N_{d o f} \times 6}^{\top}[\mathbb{C}]_{6 \times 6}[\boldsymbol{B}]_{6 \times N_{\text {dof }}}[\mathbb{D}]_{N_{\text {dof }} \times 6} d Y^{e} & =\int_{Y^{e}}[\boldsymbol{B}]_{N_{\text {dof }} \times 6}^{\top}[\mathbb{C}]_{6 \times 6} d Y^{e} \\
& =\left[\boldsymbol{F}^{D}\right]_{N_{d o f} \times 6}
\end{aligned}
$$

where the script $e$ denotes element quantities from the discretized unit-cell domain $Y .[\mathbb{C}]$ is the heterogeneous elastic stiffness matrix, $[\boldsymbol{B}]$ is the strain shape function matrix, and $[\mathbb{D}]$ is the displacement relocalization matrix whose components are solutions of Eq. (A.1). The load $\left[\boldsymbol{F}^{D}\right]$ is a matrix composed of six columns in the $3 \mathrm{D}$ cases. Each column is a force vector corresponding to an initial strain loading. To illustrate this, we recall that the nodal forces $\left\{\boldsymbol{f}^{\varepsilon^{0}}\right\}$ induced by an initial strain $\varepsilon^{0}$ are defined as:

$$
\left\{\boldsymbol{f}^{\varepsilon^{0}}\right\}=\int_{Y}[\boldsymbol{B}]^{\top}[\mathbb{C}] \boldsymbol{\varepsilon}^{0} d Y
$$

Thus, in our case, the loading are six unit initial strain tensors $E^{k l}$ applied to the unit-cell with $k l=\{11,22,33,23,31,12\}$. Indeed, Eq. (A.1) is a set of six matrix equations with six solutions, each providing a column of $[\mathbb{D}]$ :

$$
D_{i k l}=u_{i}^{1} \text { for } i \in\{1,2,3\}, k l=\{11,22,33,23,31,12\} \text {, }
$$

where $D_{i j k}$ are components of the displacement localization tensor $\mathbb{D}$. Periodic boundary conditions are imposed to the unit-cell in order to solve problems in Eq. (A.1). The resulting strain and stress fields obtained for each loading case also provide a column of the strain and stress relocalization matrices $[\mathbb{A}]$ and $[\mathbb{B}]$, respectively. The homogenized elasticity matrix $\mathbb{C}^{0}$ can be obtained from the volume average of $[\mathbb{B}]$ :

$$
\left[\mathbb{C}^{0}\right]=\frac{1}{|Y|} \int_{Y}[\mathbb{B}] d Y
$$


Practically, the components of $\mathbb{C}^{0}$ can be computed by solving elementary load cases corresponding to the different components of the macroscale strain $E^{k l}$ and performing a unit-cell average of the resulting microscopic stress components.

Flowchart for the numerical resolution of $\left(\mathcal{P}_{\text {order }}^{\mathcal{1}^{\text {st }}}\right)$

In practice, after discretizing the unit-cell domain, it is sufficient to run the finite element program for six different unit initial strain tensors $E^{k l}$ applied on the unit-cell. With $k l=\{11,22,33,23,31,12\}$ each load case provides a column vector of $[\mathbb{D}],[\mathbb{A}]$, and $[\mathbb{B}]$. Components of the displacement corrector matrix $[\mathbb{D}]$ are stored at the nodes and those of the relocalization matrices $[\mathbb{A}]$ and $[\mathbb{B}]$ are stored at integration points. The main steps for the numerical resolution of the first-order problem are summarized in Fig. A.1. 


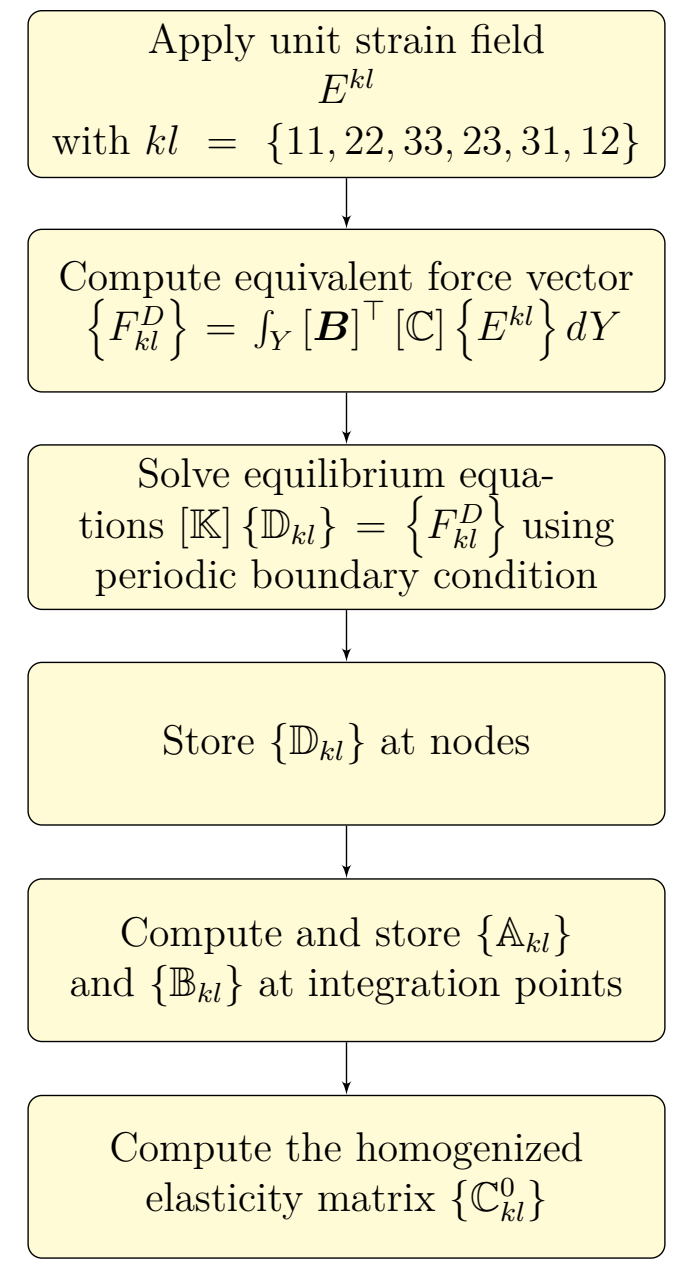

Figure A.1: Flowchart summarizing the numerical resolution of $\left(\mathcal{P}_{\text {order }}^{1^{\text {st }}}\right)$. 


\section{Appendix B: Uni-directional laminated composite in tension}

We consider a uni-directional laminated composite made of two layers as presented in Fig. B.1. The size of the structure is $L=8 \mathrm{~mm}, H=5 \mathrm{~mm}$ and $W=$ $1 \mathrm{~mm}$. The two layers are assumed to be isotropic linear elastic with coefficients $\left(E_{m}=1000 \mathrm{MPa}, \nu_{m}=0.3\right)$ and $\left(E_{f}=10 E_{m}, \nu_{f}=0.3\right)$.

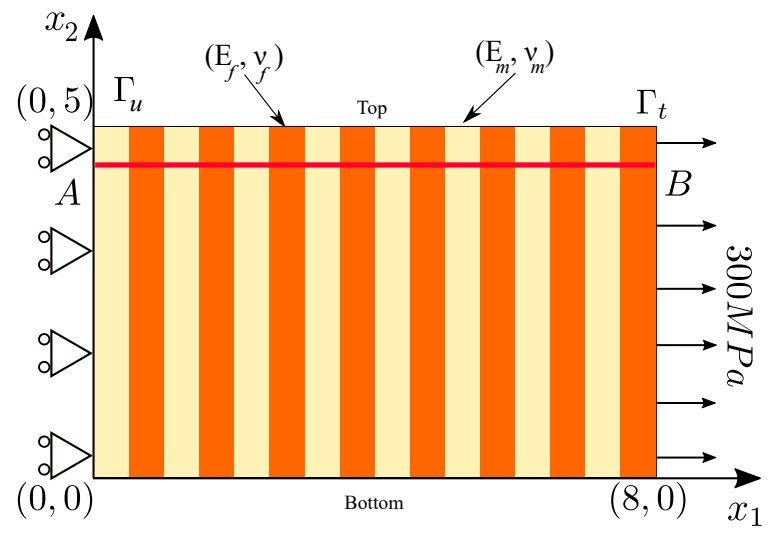

(a) Structure geometry

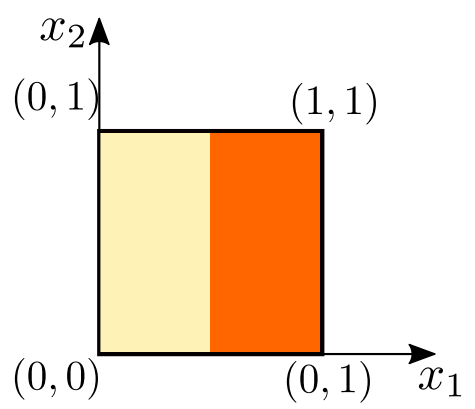

(b) unit-cell domain

Figure B.1: Illustration of the uni-directional laminated composite in tension. The structure is sliding on $\Gamma_{u}$ and a surface tension is applied on $\Gamma_{t}$.

\section{Case 1: Flat Top and Bottom surfaces}

In this case, periodicity conditions are applied to Top and Bottom surfaces. Both homogeneous and heterogeneous solution fields depend only on the $x_{1}$ variable. Estimated fields using relocalization process are supposed to match exactly with the heterogeneous ones, even on the boundaries as there is no fluctuation of material properties in the $x_{2}$ direction (see $\varepsilon_{11}^{r e f}, \varepsilon_{11}^{\text {hom }}$ and $\varepsilon_{11}^{\text {est }}$ in Fig. B.2). Therefore, no boundary layer correction is needed.

\section{Case 2: Free Top and Bottom surfaces}

In this case, heterogeneous fields are expected to fluctuate at Top and Bottom surfaces due to the heterogeneity of the material along these free edges. Therefore, a boundary layer correction in these surfaces is necessary in order to correctly estimate heterogeneous fields (see $\sigma_{11}^{\text {ref }}, \sigma_{11}^{\text {est }}$ and $\sigma_{11}^{\text {cor }}$ in Fig. B.2).

Results of stress fields are plotted in Fig. B.3 along the AB line showed in Fig. B.1. The corrected stress field $\sigma_{11}^{\text {cor }}$ captures the fluctuation of the reference field $\sigma_{11}^{\text {ref }}$ contrary to the estimated field $\sigma_{11}^{\text {est }}$ which is constant. The difference between relocalized 
and corrected fields at $x_{1}=[0,1]$ and $x_{1}=[7,8]$ in Fig. B.3 is due to the imperfection of the proposed correction for corner cells.
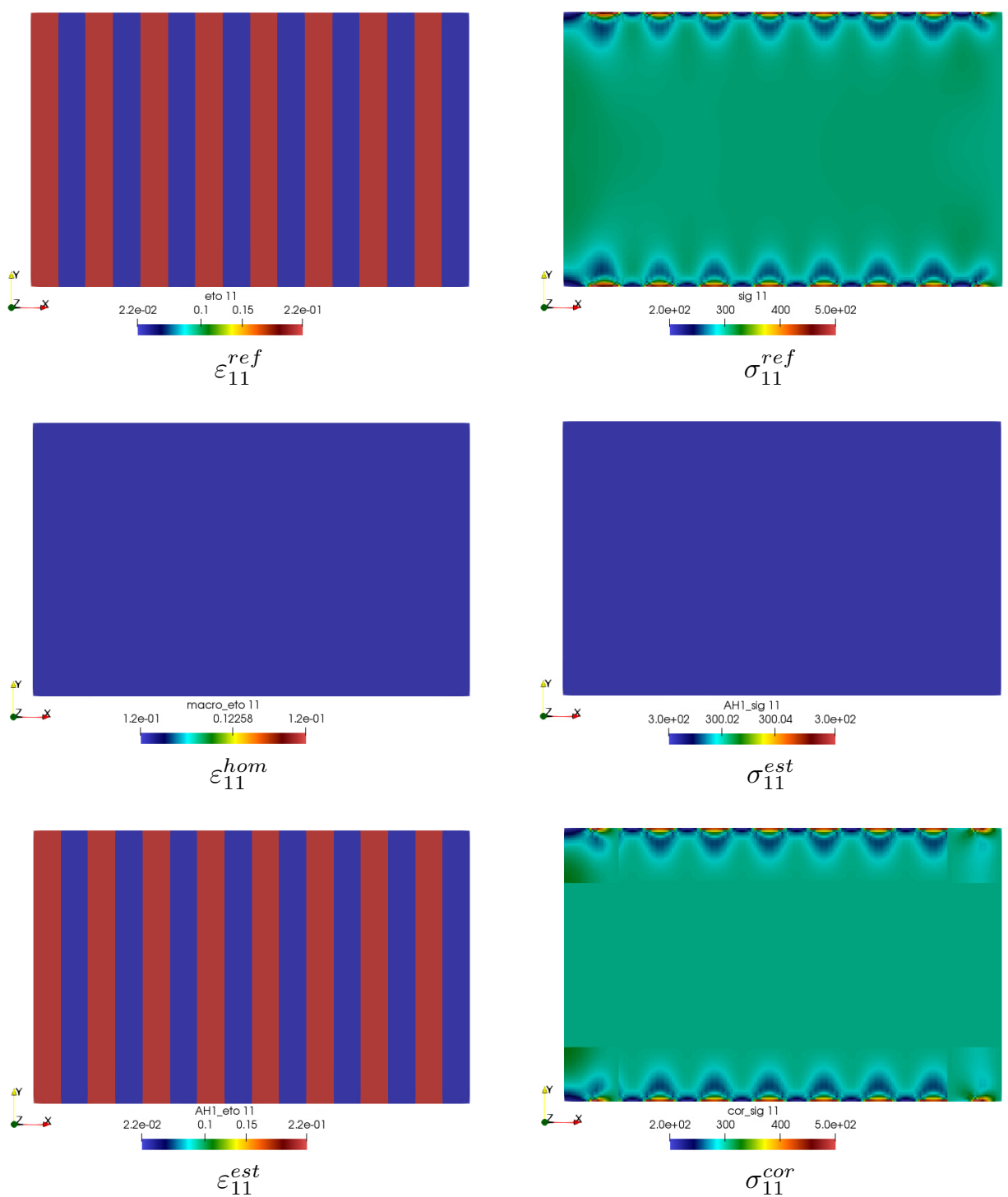

Figure B.2: Results of the strain fields for case 1 (Periodicity conditions on Top and Bottom surfaces) and stress fields for case 2 (Free Top and Bottom surfaces). 


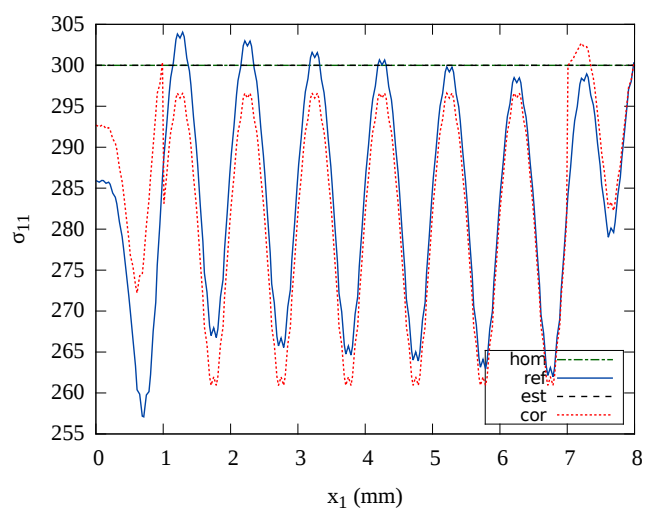

(a) $\sigma_{11}(\mathrm{MPa})$

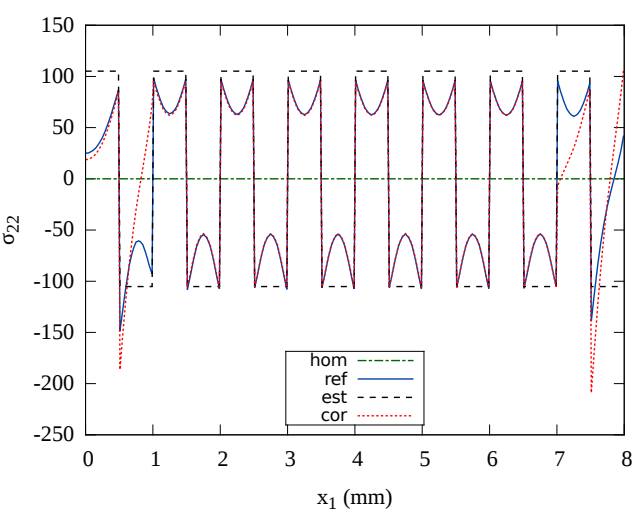

(b) $\sigma_{22}(\mathrm{MPa})$

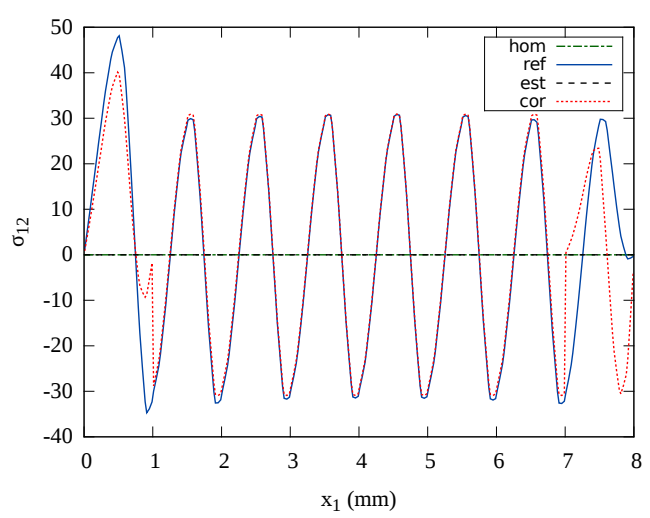

(c) $\sigma_{12}(\mathrm{MPa})$

Figure B.3: Results of the homogenized (hom), reference (ref), estimated (est) and corrected (cor) stress fields corresponding to case 2 and plotted along the line AB. 


\section{References}

[1] W. Voigt, Ueber die Beziehung zwischen den beiden Elasticitätsconstanten isotroper Körper, Annalen der Physik 274 (12) (1889) 573-587.

[2] A. Reuß, Berechnung der Fließgrenze von mischkristallen auf grund der Plastizitätsbedingung für Einkristalle., ZAMM-Journal of Applied Mathematics and Mechanics/Zeitschrift für Angewandte Mathematik und Mechanik 9 (1) (1929) 49-58.

[3] Z. Hashin, S. Shtrikman, A variational approach to the theory of the elastic behaviour of multiphase materials, Journal of the Mechanics and Physics of Solids 11 (2) (1963) 127-140.

[4] R. Hill, A self-consistent mechanics of composite materials, Journal of the Mechanics and Physics of Solids 13 (4) (1965) 213-222.

[5] T. Mori, K. Tanaka, Average stress in matrix and average elastic energy of materials with misfitting inclusions, Acta metallurgica 21 (5) (1973) 571-574.

[6] S. Ghosh, K. Lee, S. Moorthy, Multiple scale analysis of heterogeneous elastic structures using homogenization theory and voronoi cell finite element method, International Journal of Solids and Structures 32 (1) (1995) 27-62.

[7] F. Feyel, J.-L. Chaboche, FE2 multiscale approach for modelling the elastoviscoplastic behaviour of long fibre $\mathrm{SiC} / \mathrm{Ti}$ composite materials, Computer methods in applied mechanics and engineering 183 (3-4) (2000) 309-330.

[8] K. Terada, N. Kikuchi, A class of general algorithms for multi-scale analyses of heterogeneous media, Computer methods in applied mechanics and engineering 190 (40-41) (2001) 5427-5464.

[9] M. Ameen, R. Peerlings, M. Geers, A quantitative assessment of the scale separation limits of classical and higher-order asymptotic homogenization, European Journal of Mechanics - A/Solids 71 (2018) 89-100.

[10] V. Kouznetsova, M. Geers, W. Brekelmans, Multi-scale second-order computational homogenization of multi-phase materials: a nested finite element solution strategy, Computer Methods in Applied Mechanics and Engineering 193 (48-51) (2004) 5525-5550. 
[11] X. Yuan, Y. Tomita, T. Andou, A micromechanical approach of nonlocal modeling for media with periodic microstructures, Mechanics Research Communications 35 (1-2) (2008) 126-133.

[12] S. Forest, D. Trinh, Generalized continua and non-homogeneous boundary conditions in homogenisation methods, ZAMM - Journal of Applied Mathematics and Mechanics / Zeitschrift für Angewandte Mathematik und Mechanik 91 (2) (2011) 90-109.

[13] J. Yvonnet, N. Auffray, V. Monchiet, Computational second-order homogenization of materials with effective anisotropic strain-gradient behavior, International Journal of Solids and Structures 191-192 (2020) 434-448.

[14] E. Sanchez-Palencia, Homogenization method for the study of composite media, in: Asymptotic Analysis II-, Springer, 1983, pp. 192-214.

[15] A. Bensoussan, J.-L. Lions, G. Papanicolaou, Asymptotic analysis for periodic structures, Vol. 374, American Mathematical Soc., 2011.

[16] C. Boutin, Microstructural effects in elastic composites, International Journal of Solids and Structures 33 (7) (1996) 1023-1051.

[17] V. Monchiet, N. Auffray, J. Yvonnet, Strain-gradient homogenization: A bridge between the asymptotic expansion and quadratic boundary condition methods, Mechanics of Materials 143 (2020) 103309.

[18] O. Sigmund, Tailoring materials with prescribed elastic properties, Mechanics of Materials 20 (4) (1995) 351-368.

[19] K. Suzuki, N. Kikuchi, A homogenization method for shape and topology optimization, Computer Methods in Applied Mechanics and Engineering 93 (3) (1991) 291-318.

[20] B. Hassani, E. Hinton, A review of homogenization and topology opimization ii - analytical and numerical solution of homogenization equations, Computers \& structures 69 (6) (1998) 719-738.

[21] P. W. Chung, K. K. Tamma, Woven fabric composites - developments in engineering bounds, homogenization and applications, International Journal for Numerical Methods in Engineering 45 (12) (1999) 1757-1790. 
[22] A. Visrolia, M. Meo, Multiscale damage modelling of 3D weave composite by asymptotic homogenisation, Composite Structures 95 (2013) 105-113.

[23] H. Moulinec, P. Suquet, A fast numerical method for computing the linear and nonlinear mechanical properties of composites, Comptes rendus de l'Académie des sciences. Série II. Mécanique, physique, chimie, astronomie. (1994).

[24] J. C. Michel, H. Moulinec, P. Suquet, A computational method based on augmented lagrangians and Fast Fourier transforms for composites with high contrast, CMES(Computer Modelling in Engineering \& Sciences) 1 (2) (2000) 79 88.

[25] T.-H. Tran, V. Monchiet, G. Bonnet, A micromechanics-based approach for the derivation of constitutive elastic coefficients of strain-gradient media, International Journal of Solids and Structures 49 (5) (2012) 783-792.

[26] P. W. Chung, K. K. Tamma, R. R. Namburu, Asymptotic expansion homogenization for heterogeneous media: computational issues and applications, Composites Part A: Applied Science and Manufacturing 32 (9) (2001) 1291-1301.

[27] G.-D. Cheng, Y.-W. Cai, L. Xu, Novel implementation of homogenization method to predict effective properties of periodic materials, Acta Mechanica Sinica 29 (4) (2013) 550-556.

[28] J. Oliveira, J. Pinho-da Cruz, F. Teixeira-Dias, Asymptotic homogenisation in linear elasticity. Part II: Finite element procedures and multiscale applications, Computational Materials Science 45 (4) (2009) 1081-1096.

[29] T. A. Dutra, R. T. L. Ferreira, H. B. Resende, A. Guimarães, J. M. Guedes, A complete implementation methodology for Asymptotic Homogenization using a finite element commercial software: preprocessing and postprocessing, Composite Structures 245 (2020) 112305.

[30] R. B. Pipes, B. Kaminski, N. Pagano, Influence of the free edge upon the strength of angle-ply laminates, ASTM International, 1973.

[31] S. Tang, A. Levy, A boundary layer theory-part ii: extension of laminated finite strip, Journal of Composite Materials 9 (1) (1975) 42-52.

[32] P. W. Hsu, C. T. Herakovich, Edge effects in angle-ply composite laminates, Journal of Composite Materials 11 (4) (1977) 422-428. 
[33] N. J. Pagano, Free edge stress fields in composite laminates, International Journal of Solids and Structures 14 (5) (1978) 401-406.

[34] E. Sanchez-Palencia, Homogenization in mechanics. a survey of solved and open problems, Rend. Sem. Mat. Univ. Politec. Torino 44 (1) (1986) 1-45.

[35] H. Dumontet, Study of a boundary layer problem in elastic composite materials, ESAIM: Mathematical Modelling and Numerical Analysis 20 (2) (1986) 265-286.

[36] M. Lefik, B. Schrefler, FE modelling of a boundary layer corrector for composites using the homogenization theory, Engineering computations (1996).

[37] R. Abdelmoula, A. Leger, Local and global effects of small holes periodically distributed on a surface embedded in an axisymmetrical elastic medium, European Journal of Mechanics-A/Solids 24 (1) (2005) 89-109.

[38] S. Koley, P. Mohite, C. Upadhyay, Boundary layer effect at the edge of fibrous composites using homogenization theory, Composites Part B: Engineering 173 (2019) 106815.

[39] B. Hassani, E. Hinton, Homogenization and Structural Topology Optimization, Springer London, London, 1999.

[40] S. Kruch, Homogenized and relocalized mechanical fields, The Journal of Strain Analysis for Engineering Design 42 (4) (2007) 215-226.

[41] S. Kruch, S. Forest, Computation of coarse grain structures using a homogeneous equivalent medium, Journal de Physique IV 08 (PR8) (1998) Pr8-197-Pr8-205. 